\title{
Various applications of analog signal processing employing voltage differencing current conveyor and only grounded passive elements: a re-convertible approach
}

\author{
Manish Gupta ${ }^{1} \cdot$ Tajinder Singh Arora $^{2}$ (I)
}

Received: 12 December 2019 / Accepted: 19 August 2020 / Published online: 1 September 2020

(c) Springer Nature Switzerland AG 2020

\begin{abstract}
A re-convertible circuit that can either act as a current-mode universal filter or as a fully un-coupled dual-mode singleresistance-controlled quadrature oscillator or simply works as a grounded loss-less inductor, by simple selection of appropriate passive switches, is proposed here. The proposed circuit requires a maximum of two voltage differencing current conveyors as an active element in the case of filter and oscillator application, whereas only one active device suffices the purpose of active inductor implementation. In all the cases only grounded passive elements are employed. Various regular analysis for the proposed circuits have also been included here to support the theoretical idea. The workability of these circuits has been demonstrated by practicing PSPICE simulation software with TSMC CMOS $0.18 \mu \mathrm{m}$ process parameters along with the experimental justification of the derived application using commercially available integrated circuits.
\end{abstract}

Keywords Analog signal processing · Active filter · Single-resistance-controlled-oscillator · Grounded inductor · Current mode circuits · Universal filter

\section{Introduction}

Analog signal processing is about the conditioning of natural signals. Despite the advancements in digital signal processing, analog signal processing has its own space as all the natural signals are analog in nature. Realization of active filters, sinusoidal oscillators, and synthetic inductors attracted the researchers due to their different applications in communication, signal processing, and control engineering as a signal generator, frequency selective filters, etc. $[1,2]$.

The design solutions for analog signal processing applications by employing different active building blocks such as the realization of a synthetic inductor [3-8], formation of active filters [9-21], and generation of sinusoidal oscillators [19, 21-33] are available in the open literature. Inductors can be further classified as grounded [3-7] and ungrounded or floating inductors $[6,8]$. On the other hand, active filters can also be classified as current-mode (whereas input and output both are the functions of current only) [9-11, 14-19, 21 , voltage mode (when the input and output both are voltages) $[12,13,20]$, trans-impedance mode (when the current is fed at the input of the circuit and voltage is obtained as an output) and trans-conductance mode (when the output is in the form of current and voltage is the input to the circuit) $[11,12,15]$. In sinusoidal oscillators single phase output $[27,30]$ and multiphase outputs $[19,21-26,28,29,31-33]$ can be the major criterion to divide the other class of oscillators. Quadrature oscillators are being a special class of multiphase oscillators that can also be classified as voltage-mode

$\triangle$ Tajinder Singh Arora, tsarora@nituk.ac.in | ${ }^{1}$ Department of Electronics and Communication Engineering, Inderprastha Engineering College, Ghaziabad, Uttar Pradesh, India. ${ }^{2}$ Department of Electronics Engineering, National Institute of Technology Uttarakhand, Srinagar, India. 
$[26,29,31]$, current-mode $[19,23,32]$, and dual-mode circuits $[21,22,28,33]$.

Voltage differencing current conveyor (VDCC) is a versatile active block that was first investigated by Biolek et al. [34]. Since then, the device has been actively used in designing and realizations of analog signal processing applications. However, being such an eminent active device, it is yet to be explored to its full potential. This is the major reason behind keeping the said device under focus, in this manuscript.

The purpose of this manuscript is to present one such circuit that possesses the following characteristics, simultaneously.

1. The circuit can also be configured into a grounded loss-less synthetic inductor.

2. When acting as a filter, the circuit behaves as SIMO (single input multiple output) type current-mode (CM) biquad with low input impedance and high output impedance. Additionally, it offers the availability of explicit current output and independent tunability of its quality factor.

3. When made to operate as a sinusoidal oscillator, it provides quadrature outputs in both current as well as voltage modes (VM). Generation of explicit current outputs, with simple and uncoupled condition of oscillation (CO) and frequency of oscillation (FO) are few appreciated features.

All the above-mentioned applications can be obtained through the derived circuit with the help of a combination of passive switches. All the proposed applications utilize only grounded passive elements, a minimum number of active devices, and are least affected by the parasitic of the used active element. The comparison of the proposed work, i.e., grounded synthetic inductor, active filter, and quadrature sinusoidal oscillator, with the existing literature is discussed in detail in Sect. 6.

This manuscript has been divided into various sections, wherein, each section provides a detailed description of the work. Section 2 gives the introduction of the active device, i.e., VDCC. The proposed circuit is described in Sect. 3. Section 4 presents the non-ideal analysis of all the proposed applications. The sensitivity analysis of all the designs is represented in Sect. 5. Section 6 represents a detailed and comprehensive literature survey. Simulation results practicing PSPICE software are given in Sect. 7. Experimental work has been performed using off-the-shelf integrated circuits and is provided in Sect. 8. Concluding remarks have been presented at last.

\section{Introduction to VDCC}

VDCC is a versatile active element that is an electrical combination of OTA and CCII. This active element has the adeptness of conveying the current from one direction to other and simultaneously providing the voltage buffered input terminals. The block diagram of the VDCC is given in Fig. 1.

The active device has in total six terminals namely $\mathrm{P}, \mathrm{N}$, $\mathrm{Z}, \mathrm{X}, \mathrm{W}_{\mathrm{P}}$, and $\mathrm{W}_{\mathrm{N}}$. Out of these six terminals, two input terminals $P$ and $N$ are high impedance ports, three output terminals $Z, W_{p}$ and $W_{N}$ are high impedance ports and $X$ is a low impedance output port. As it has been quoted earlier that $C C$ is an inherent part of this device so by exploiting the nature of $\mathrm{CC}$ multiple copies of the $\mathrm{Z}$ terminal currents can also be obtained and marked as $Z c+$ and $Z c$ - depending upon its direction of flow. The terminal equations of VDCC are presented by (1). For simulation purposes, a CMOS (complementary metal oxide semiconductor) version of VDCC [7] is being used, as given in Fig. 2. For hardware realization of VDCC, a commercially available integrated circuit, i.e., OPA860 has been used and it is discussed in detail in Sect. 8. The circuit diagram of VDCC, when implemented using OPA860 and few passive elements, is shown in Fig. 3, which is a modified version of [28]. Basically the latest version of the VDCC architecture is proposed in [28] and this is a corrected version of [20].

$\left[\begin{array}{l}I_{N} \\ I_{P} \\ I_{Z} \\ V_{X} \\ I_{W P} \\ I_{W N}\end{array}\right]=\left[\begin{array}{cccc}0 & 0 & 0 & 0 \\ 0 & 0 & 0 & 0 \\ g_{m} & -g_{m} & 0 & 0 \\ 0 & 0 & 1 & 0 \\ 0 & 0 & 0 & 1 \\ 0 & 0 & 0 & -1\end{array}\right]\left[\begin{array}{l}V_{P} \\ V_{N} \\ V_{Z} \\ I_{X}\end{array}\right]$

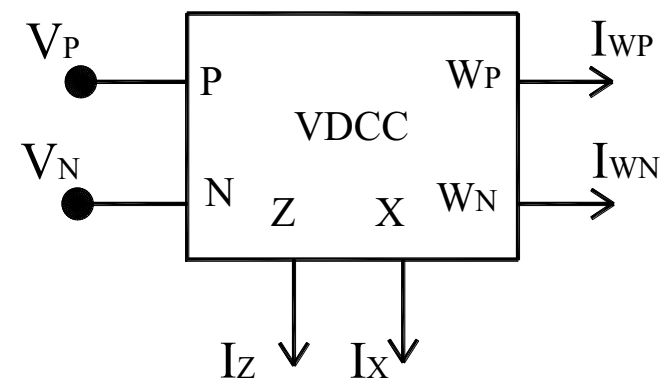

Fig. 1 Block diagram of VDCC [7] 


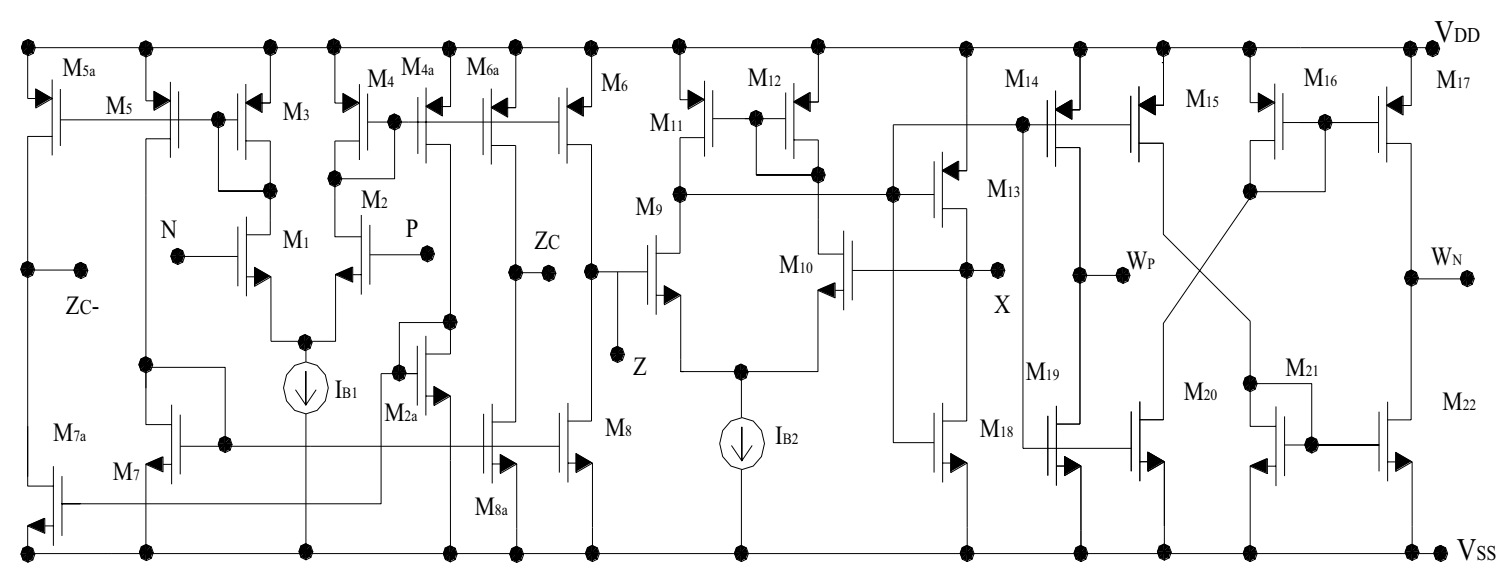

Fig. 2 CMOS realization of the voltage differencing current conveyor [7]

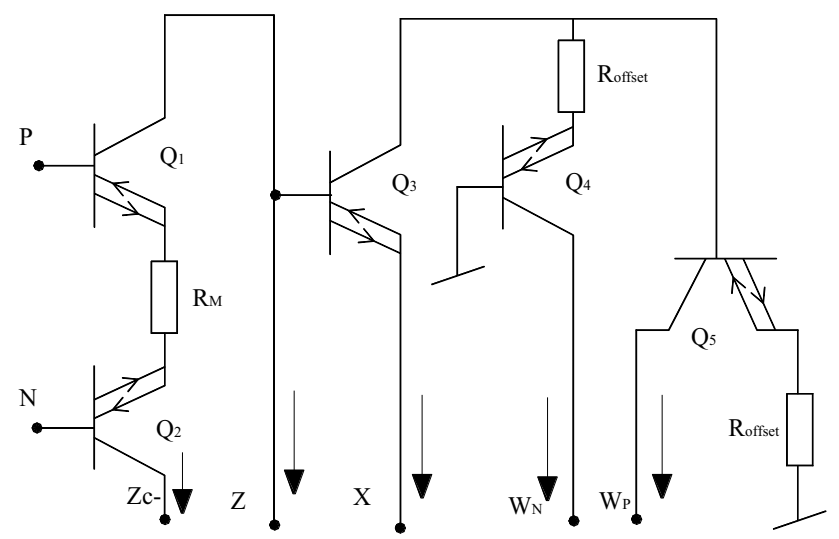

Fig. 3 Realization of VDCC utilizing discrete components [28]

\section{Proposed re-convertible circuit}

In the sphere of analog signal processing, continuous-time filters, active inductor realizations, and single-resistance-controlled-oscillators preoccupy a special space. The proposed reconfigurable circuit is capable of acting as an active inductor, continuous time secondorder universal filter and dual mode quadrature oscillator by changing the direction of the passive switches. The proposed circuit with only grounded passive elements and VDCC is shown in Fig. 4.

For the realization of different applications using the designed circuit (as shown in Fig. 4), an appropriate passive switch combination must be applied which is represented in Table 1.

\subsection{Realization of grounded loss-less inductor}

For inductor realization, the switch combination given at S. No. 1 of Table 1 is applied in Fig. 4. Here, for simplicity, the voltage input $\left(\mathrm{V}_{\mathrm{IN}}\right)$ and current through this $\left(\mathrm{I}_{\mathrm{IN}}\right)$ are omitted. When $V_{\mathbb{I N}}$ is applied at the end of the switch S4, the routine circuit analysis and simple circuit theory calculations generate the input impedance of the circuit, as given in (2). While the equivalent inductance is shown
Fig. 4 A re-convertible proposed circuit using VDCC

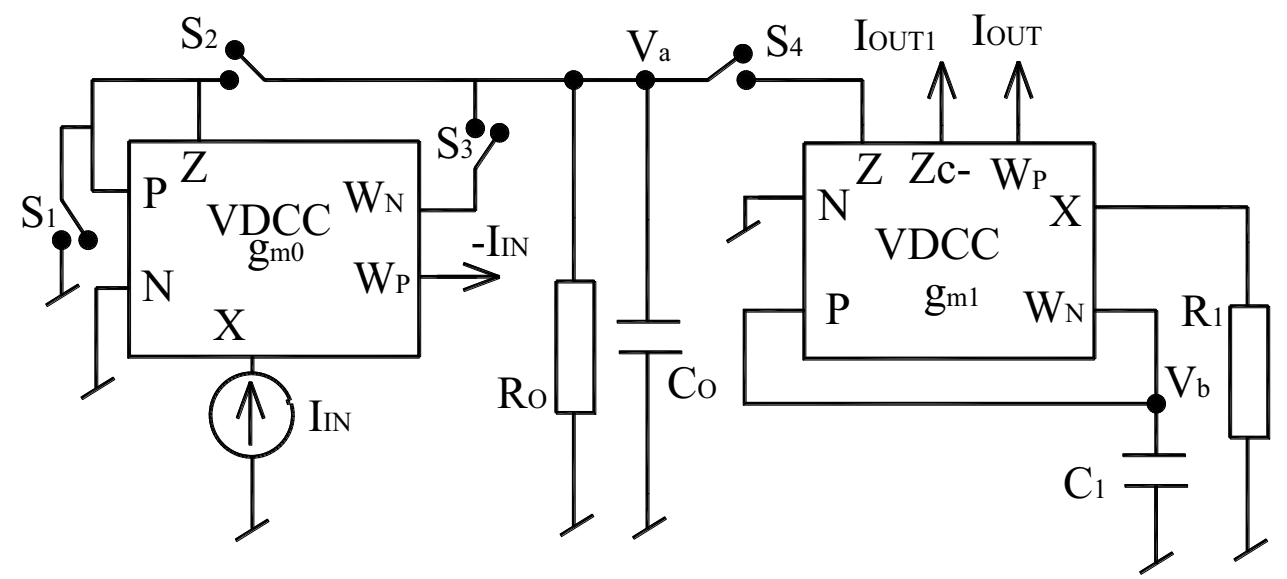


Table 1 Characteristic table (for the circuit shown in Fig. 4)

\begin{tabular}{llllll}
\hline S. no. & \multicolumn{3}{l}{ Passive switches } & & Operation \\
\cline { 2 - 4 } & S1 & S2 & S3 & S4 & \\
\hline 1. & - & - & - & OFF & Active inductor realization \\
2. & ON & OFF & ON & ON & Current mode universal filter \\
3. & OFF & ON & OFF & ON & Dual mode quadrature oscillator \\
\hline
\end{tabular}

by (3). The designed grounded lossless inductor is either electronically tunable by $g_{m 1}$ or controllable by grounded resistor $\mathrm{R}_{1}$, as represented in (3).

$\frac{V_{I N}}{I_{I N}}=Z_{I N}=\frac{s C_{1} R_{1}}{g_{m 1}}=s \cdot L_{e q}$

$L_{e q}=\frac{C_{1} R_{1}}{g_{m 1}}$

\subsection{Realization of current mode universal filter}

When the switch combination of S. No. 2 (Table 1) is applied in Fig. 4, the outcome of the configuration is a universal current mode second order continuous time filter. This biquadratic filter has three explicit current outputs namely $I_{\text {OUT }}\left(I_{B P}\right), I_{\text {OUT1 }}\left(I_{L P}\right)$, and $I_{I N}$ with a negative sign. Obtaining the high pass filter response requires an appropriate combination of these three explicit currents. Here it is worth noting that the input impedance, where the current is being injected, is zero which is a desirable feature of the current-mode active filters. The transfer functions for band pass, high pass, and low pass filter functions are given in (4)-(6). The common denominator polynomial, angular frequency, and quality factor are represented by (7) to (9) respectively. It can be seen from (9) that the quality factor of the designed filter is independently tunable with the help of $R_{0}$ without changing its center frequency.

$$
\frac{I_{B P}}{I_{I N}}=\frac{\frac{s}{R_{1} C_{0}}}{D(s)}
$$

$\frac{I_{L P}}{I_{I N}}=\frac{\frac{g_{m 1}}{R_{1} C_{0} C_{1}}}{D(s)}$

$$
\text { If } R_{1}=R_{0}
$$

$\frac{I_{H P}}{I_{I N}}=\frac{I_{I N}-I_{B P}-I_{L P}}{I_{I N}}=\frac{s^{2}}{D(s)}$
$D(s)=s^{2}+\frac{s}{R_{0} C_{0}}+\frac{g_{m 1}}{R_{1} C_{0} C_{1}}$

$\omega_{0}=\sqrt{\frac{g_{m 1}}{R_{1} C_{0} C_{1}}}$

$Q_{0}=R_{0} \sqrt{\frac{g_{m 1 C_{0}}}{R_{1} C_{1}}}$

\subsection{Realization of dual mode quadrature oscillator}

Dual-mode single-resistance-controlled-quadrature oscillator, employing VDCC and all grounded passive elements, can also be derived from Fig. 4 when the desirable switch sequence is applied (as given in Table 1 at S. No. 3). The proposed oscillator has uncoupled $\mathrm{CO}$ and $\mathrm{FO}$ along with the availability of quadrature outputs in current as well as in voltage representation. Two explicit current output terminals are $\mathrm{I}_{\text {OUT }}$ and $\mathrm{I}_{\text {OUT } 1}$. The characteristic equation of the designed oscillator is given in (10). Whereas, the $\mathrm{CO}$ and $\mathrm{FO}$ are represented by (11) and (12) respectively. The expression for the quadrature output in both the modes is given in (13).

$s^{2}+\frac{s}{C_{0}}\left(\frac{1}{R_{0}}-g_{m 0}\right)+\frac{g_{m 1}}{R_{1} C_{0} C_{1}}=0$

C.O. $\left(\frac{1}{R_{0}}-g_{m 0}\right) \geq 0$

F.O. $\omega_{0}=\sqrt{\frac{g_{m 1}}{R_{1} C_{0} C_{1}}}$

$\frac{I_{\text {OUT } 1}}{I_{\text {OUT }}}=-\frac{g_{m 1}}{s C_{1}}$ and $\frac{V_{\text {OUT } 1}}{V_{\text {OUT }}}=-\frac{1}{s C_{1} R_{1}}$

It can be easily seen in (11) and (12) that $\mathrm{CO}$ can be easily adjusted with the help of grounded resistor $\mathrm{R}_{0}$ or can be electronically tuned through trans-conductance factor $\mathrm{g}_{\mathrm{m} 0}$. To achieve the variation in the frequency of oscillation, a grounded resistor $R_{1}$ can be adjusted or electronic tunability can be achieved from $g_{m 1}$. Further in (12) if $g_{m 1}=1 / R_{1}$ and $C_{0}=C_{1}$, the frequency of oscillations can also be tuned linearly, which further enhances the feature of the designed oscillator. 


\section{Non-ideal analysis including the influence of parasitic}

To check the deviation between ideal and non-ideal responses of the proposed circuits, device non-idealities and parasitic have been taken into consideration. The nonideal characteristics equations of VDCC [21] is represented by (14). The non-ideal factors are defined as $\alpha, \beta, \gamma_{P}$ and $\gamma_{N}$. The ideal value of these factors is unity.

$$
\left.\begin{array}{l}
I z=\alpha \cdot g_{m}\left(V_{P}-V_{N}\right) \\
V_{Z}=\beta \cdot V_{X} \\
I_{W P}=\gamma_{P} I_{X} \\
I_{W N}=-\gamma_{N} I_{X}
\end{array}\right\}
$$

A widely agreeable parasitic model of VDCC has been taken from [21], and redrawn in Fig. 5.

When both the non-ideal effects, i.e., accouterment of Fig. 5 and non-ideal gain effects of (14) are considered together on the proposed circuit, its mathematical outcome has been provided in (15-26).

Figure 6 shows the synthetic inductor configuration under the effect of both non-ideal contributors. The ratio between the input voltage and the input current is reflected in (16). In order to derive (16) some assumptions have been taken into account, as shown in (15).

Assumptions

$$
\left.\begin{array}{l}
R_{1} \gg R_{X} \\
C_{1}>C_{N}
\end{array}\right\}
$$

$$
Z_{I N}=\frac{\beta \cdot R_{1} R_{Z}\left(s C_{1} R_{N}+1\right)}{\left(s C_{1} R_{N}+1\right) \beta \cdot R_{1}+\alpha \cdot \gamma_{N} \cdot g_{m 1} R_{N} R_{Z}}
$$

Figure 7 shows the design of the current-mode universal filter under the impact of both the non-ideal factors.

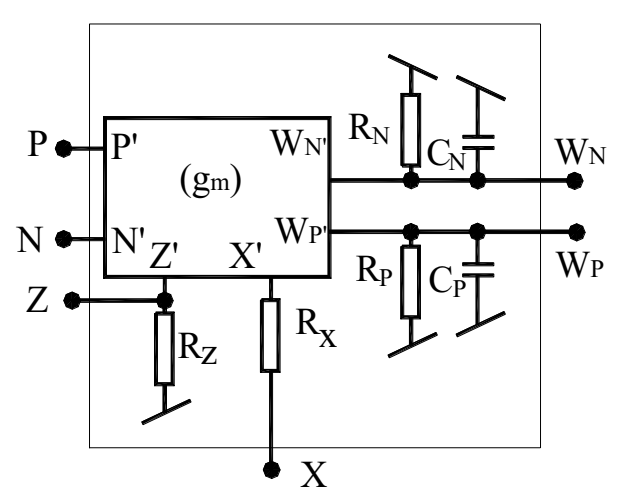

Fig. 5 VDCC including device parasitic [21]

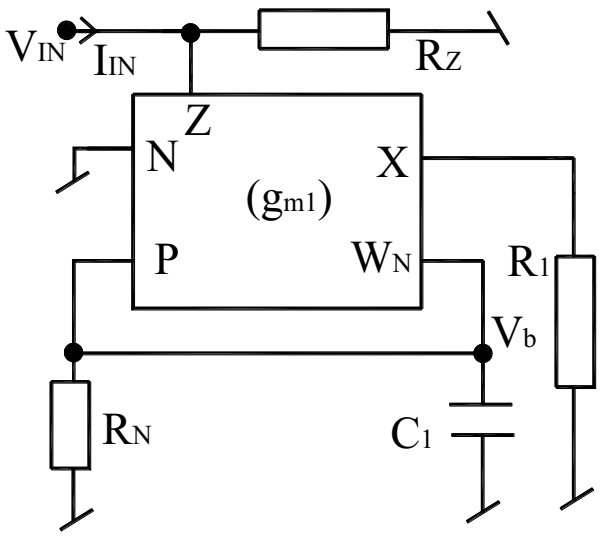

Fig. 6 Synthetic grounded inductor under the influence of parasitic

The assumptions to consolidate the transfer functions, for band pass and low pass respectively as shown in (18) and (19), is represented by (17). The common non-ideal denominator polynomial is given by (20). The non-ideal frequency and the quality factor are given by (21) and (22) respectively. It is worth noting here that the HP response will be severely affected by the non-idealities because of the cumulative effect of both the filter functions (including non-idealities) i.e., LP and BP will come into the effect.

$\left.\begin{array}{l}R_{0}<<R_{Z} \| R_{N 1} \\ R_{1}>>R_{X} \\ C_{0}>>C_{N 1} \\ C_{1}>>C_{N 2}\end{array}\right\}$

$\frac{I_{B P}}{I_{I N}}=\frac{\gamma_{P} \gamma_{N}}{\beta} \frac{\left(s C_{1} R_{N}+1\right)}{C_{0} C_{1} R_{1} R_{N}} \cdot \frac{1}{D^{\prime \prime}(s)}$

$\frac{I_{L P}}{I_{I N}}=\left[\frac{\alpha \gamma_{N}^{2}}{\beta}\right] \frac{g_{m 1}}{C_{0} C_{1} R_{1}} \cdot \frac{1}{D^{\prime \prime}(s)}$

$D^{\prime \prime}(s)=s^{2}+s\left(\frac{1}{C_{1} R_{N}}+\frac{1}{C_{0} R_{0}}\right)+\left(\frac{1}{C_{0} R_{0} C_{1} R_{N}}+\frac{\alpha \gamma_{N}}{\beta} \frac{g_{m 1}}{C_{0} C_{1} R_{1}}\right)=0$

$\omega_{0}^{\prime \prime}=\sqrt{\frac{1}{C_{0} C_{1}}\left(\frac{1}{R_{0} R_{N}}+\frac{\alpha \gamma_{N}}{\beta} \frac{g_{m 1}}{R_{1}}\right)}$

$Q_{0}^{\prime \prime}=\frac{R_{0} R_{N}}{C_{0} R_{0}+C_{1} R_{N}} \sqrt{C_{0} C_{1}\left(\frac{1}{R_{0} R_{N}}+\frac{\alpha \cdot g_{m 1} \cdot \gamma_{N}}{\beta R_{1}}\right)}$

Figure 8 shows the proposed sinusoidal oscillator including the effects of the two non-ideal contributors. 
Fig. 7 CM active filter under the effect of non-idealities

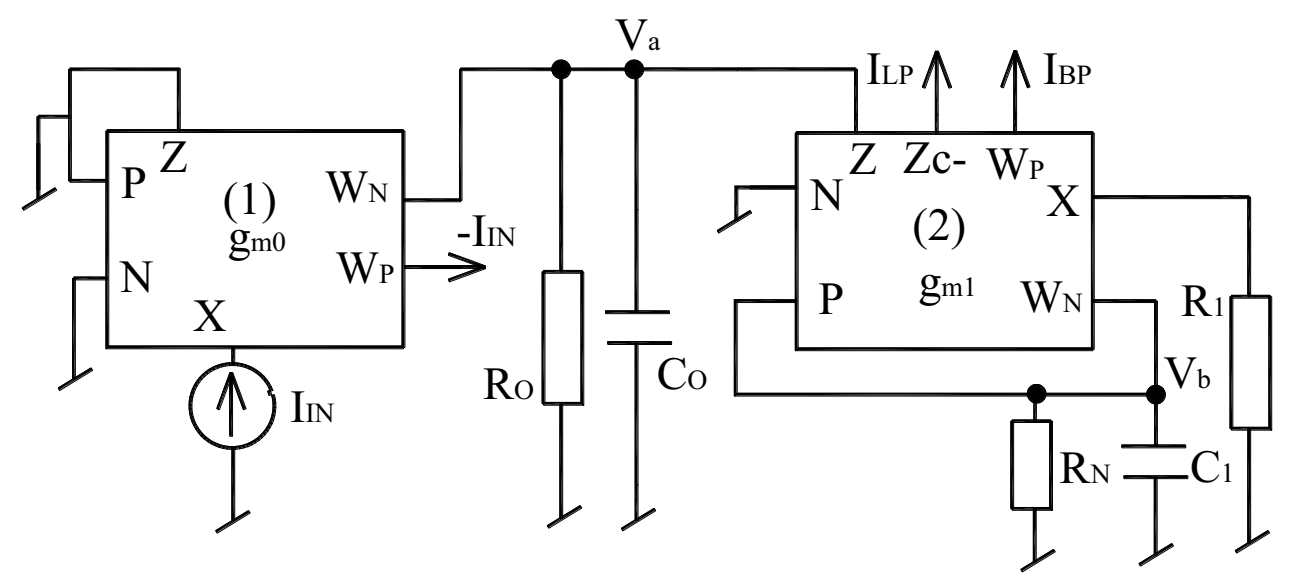

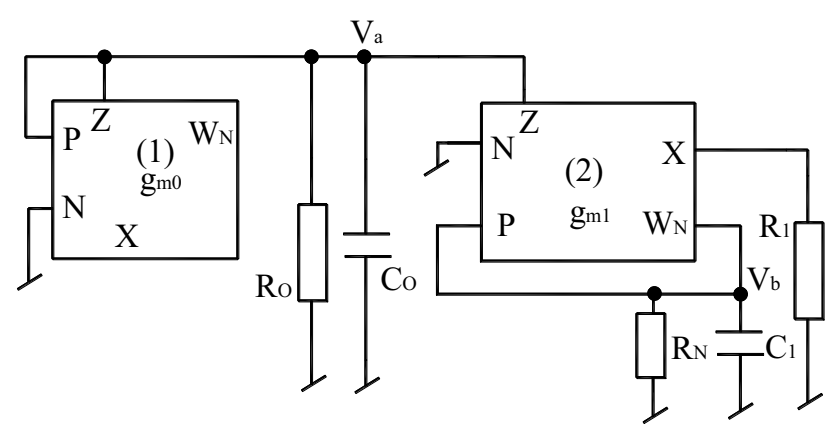

Fig. 8 Sinusoidal oscillator under the effects of non-idealities

When non-ideal effects of the active device, i.e., (14) and parasitic are applied to the designed sinusoidal oscillator circuit, the characteristic equation comes out to be as given by (24). The assumptions to find the final equations are given by (23). The mathematical equations, i.e., (25) and (26) represent the non-ideal $\mathrm{CO}$ and FO of the derived sinusoidal oscillator.

$\left.\begin{array}{l}R_{1}>>R_{X} \\ C_{0}>>C_{N 1} \\ R_{0}<<R_{Z 1}\end{array}\right\}$

$s^{2}+s\left(\frac{1}{C_{1} R_{N}}+\frac{1}{C_{0} R_{0}}-\frac{\alpha \cdot g_{m 0}}{C_{0}}\right)+\frac{1}{C_{0} C_{1}}\left(\frac{1}{R_{0} R_{N}}+\frac{\alpha \cdot g_{m 1} \gamma_{N}}{\beta \cdot R_{1}}-\frac{\alpha \cdot g_{m 0}}{R_{N}}\right)=0$

In the open literature many researchers have proposed
the design model of circuits that can act as an active filter,

In the open literature many researchers have proposed
the design model of circuits that can act as an active filter,

To reflect the impact of parasitic, on the designed oscillator, the calculation has been performed at the oscillation frequency of $1 \mathrm{MHz}$ by assuming the passive component values as $\mathrm{C}_{0}=\mathrm{C}_{1}=43.8 \mathrm{pF}, \mathrm{R}_{0}=\mathrm{R}_{1}=3.6 \mathrm{~K}$, and parasitic elements values are $R_{X}=43 \Omega, R_{Z}=362 \mathrm{~K} \Omega, R_{P}=R_{N}=141 \mathrm{~K}$ $\Omega, C_{p}=C_{N}=0.92 p F$ [21]. The oscillation frequency comes out to be $985.34 \mathrm{kHz}$ and thus shows an error of $1.5 \%$ with respect to its ideal value. It is worth noting here that the most influencing parasitic element is $R_{N}$.

\section{Sensitivity analysis}

The non-ideal active and passive sensitivity figures for all three proposed configurations, i.e., synthetic grounded loss-less inductor, current-mode universal filter and dual mode quadrature oscillator are shown in Table 2. Table 2 depicts that the entire derived sensitivity figures are under permissible limits.

\section{Comparison with existing work}

FO: $\omega_{0}^{\prime \prime}=\sqrt{\frac{1}{C_{0} C_{1}}\left(\frac{1}{R_{0} R_{N}}+\frac{\alpha \gamma_{N}}{\beta} \frac{g_{m 1}}{R_{1}}-\frac{\alpha \cdot g_{m 0}}{R_{N}}\right)}$

CO: If $\mathrm{C}_{0}=\mathrm{C}_{1} ;\left(\frac{1}{R_{0}}-g_{m 0}+\frac{1}{R_{N}}\right) \geq 0$ sinusoidal oscillator and synthetic inductor utilizing distinct methodology and employing different active building blocks (ABB) such as DVCC (differential voltage current conveyor) [9], CCII (second generation current conveyor) [15], CCIII (third generation current conveyor) [11], FDCCII (fully differential current conveyor) [12], OTA(operational trans-conductance amplifier) [18], EX-CCCII (extra copy current controlled current conveyor) [14], CCCDTA (current 
Table 2 Sensitivity figures of all three proposed applications

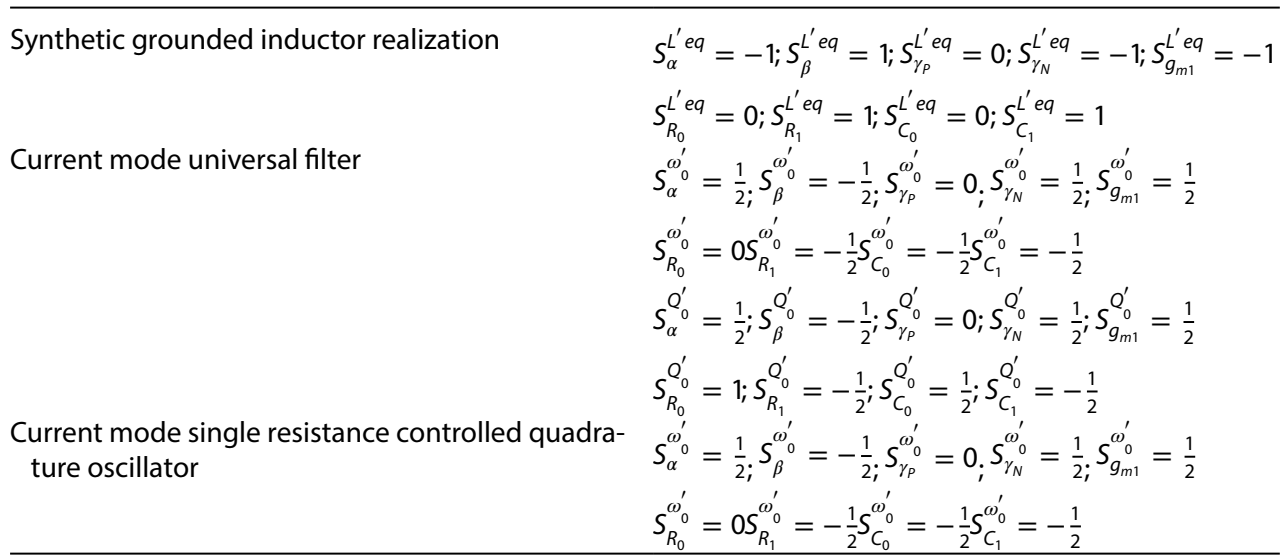

$$
\begin{aligned}
& S_{\alpha}^{L^{\prime} \text { eq }}=-1 ; S_{\beta}^{L^{\prime} \text { eq }}=1 ; S_{\gamma_{p}}^{L^{\prime} \text { eq }}=0 ; S_{\gamma_{N}}^{L^{\prime} \text { eq }}=-1 ; S_{g_{m 1}}^{L^{\prime} \text { eq }}=-1 \\
& S_{R_{0}}^{L^{\prime} \text { eq }}=0 ; S_{R_{1}}^{L^{\prime} \text { eq }}=1 ; S_{C_{0}}^{L^{\prime} \text { eq }}=0 ; S_{C_{1}}^{L^{\prime} \text { eq }}=1 \\
& S_{\alpha}^{\omega_{0}^{\prime}}=\frac{1}{2} ; S_{\beta}^{\omega_{0}^{\prime}}=-\frac{1}{2} ; S_{\gamma_{p}}^{\omega_{0}^{\prime}}=0 ; S_{\gamma_{N}}^{\omega_{0}^{\prime}}=\frac{1}{2} S_{g_{m 1}}^{\omega_{0}^{\prime}}=\frac{1}{2} \\
& S_{R_{0}}^{\omega_{0}^{\prime}}=0 S_{R_{1}}^{\omega_{0}^{\prime}}=-\frac{1}{2} S^{\omega_{0}^{\prime}} C_{0}=-\frac{1}{2} S_{C_{1}}^{\omega_{0}^{\prime}}=-\frac{1}{2} \\
& S_{\alpha}^{Q_{0}^{\prime}}=\frac{1}{2} ; S_{\beta}^{Q_{0}^{\prime}}=-\frac{1}{2} ; S_{\gamma_{p}}^{Q_{0}^{\prime}}=0 ; S_{\gamma_{N}}^{Q_{0}^{\prime}}=\frac{1}{2} ; S_{g_{m 1}}^{Q_{0}^{\prime}}=\frac{1}{2} \\
& S_{R_{0}}^{Q_{0}^{\prime}}=1 ; S_{R_{1}}^{Q_{0}^{\prime}}=-\frac{1}{2} ; S_{C_{0}}^{Q_{0}^{\prime}}=\frac{1}{2} ; S_{C_{1}}^{Q_{0}^{\prime}}=-\frac{1}{2} \\
& S_{\alpha}^{\omega_{0}^{\prime}}=\frac{1}{2} ; S_{\beta}^{\omega_{0}^{\prime}}=-\frac{1}{2} ; S_{\gamma_{p}}^{\omega_{0}^{\prime}}=0 ; S_{\gamma_{N}}^{\omega_{0}^{\prime}}=\frac{1}{2} S_{g_{m 1}}^{\omega_{0}^{\prime}}=\frac{1}{2} \\
& S_{R_{0}}^{\omega_{0}^{\prime}}=0 S_{R_{1}}^{\omega_{0}^{\prime}}=-\frac{1}{2} S_{0}^{\omega_{0}^{\prime}} C_{0}=-\frac{1}{2} S_{C_{1}}^{\omega_{0}^{\prime}}=-\frac{1}{2}
\end{aligned}
$$

controlled current differencing trans-conductance amplifier) [16], CDTA (current differencing trans-conductance amplifier) [17], MVDVTA (modified voltage differencing voltage trans-conductance amplifier) [22], CDBA (current differencing buffered amplifier) [29], VDBA (voltage differencing buffered amplifier) [33], VDIBA (voltage differencing inverting buffered amplifier) [3], CFOA (current feedback operational amplifier) [2], DO-DDCC (dual current output differential difference current conveyor) [6],VDCC (voltage differencing current conveyor) and its variants [7, $19-21,23,24,27,28]$.

Out of these mentioned references [5] employs more than 1 active device. References [4, 6] employ more than 2 passive elements whereas references $[3,6]$ do not provide both electronic and resistive tunability. A matching constraint is found in Ref. [6] and Ref. [5] do not utilize all grounded passive elements.

In comparison to the above-mentioned works, the designed synthetic inductor employs only 1 active device along with all grounded passive components. It can be tuned in both ways, i.e., electronically or through the resistive element. Moreover, there is no need of imposing a matching condition on the network in order to make it behave as an inductor.

The available features of the derived filter, i.e., employment of minimum active devices, utilization of only grounded passive elements, availability of low input impedance at the input port and high output impedance at output terminals, and independent tunability of its quality factor, cannot be simultaneously achieved in any of the paper reported in Table 3, thus justifying our design proposal. It is also very much evident from Table 4 that the said features of the quadrature oscillator, i.e., employment of the minimum number of active devices along with only grounded passive elements, provision of explicit quadrature outputs, simple and uncoupled $\mathrm{CO}$ and FO, are not

\begin{tabular}{|c|c|c|c|c|c|c|c|c|c|}
\hline S. no. & Ref. no. & $\begin{array}{l}\text { No. of } \\
\text { active } \\
\text { devices }\end{array}$ & $\begin{array}{l}\text { Name of the } \\
\text { active device }\end{array}$ & $\begin{array}{l}\text { No. of } \\
\text { passive ele- } \\
\text { ments }\end{array}$ & $\begin{array}{l}\text { Is passive } \\
\text { element } \\
\text { grounded }\end{array}$ & $\begin{array}{l}\text { Matching of } \\
\text { impedances } \\
\text { at input and } \\
\text { output level }\end{array}$ & $\begin{array}{l}\text { Is } \mathrm{Q}_{0} \text { inde- } \\
\text { pendently } \\
\text { tunnable }\end{array}$ & $\begin{array}{l}\text { Mode of } \\
\text { operation } \\
\text { (CM/VM) }\end{array}$ & $\begin{array}{l}\text { Power } \\
\text { dissipation } \\
(\mathrm{mW})\end{array}$ \\
\hline 1 & [9] & 2 & DVCC & 5 & No & No & Yes & CM & 0.81 \\
\hline 2 & [10] & 3 & CCII & 5 & Yes & No & Yes & CM & 40 \\
\hline 3 & [11] & 5 & CCIII & 9 & Yes & Yes & Yes & CM & - \\
\hline 4 & [12] & 2 & FDCCII & 6 & No & No & Yes & VM & 1.32 \\
\hline 5 & [13] & 6 & OTA & 2 & Yes & Yes & Yes & VM & - \\
\hline 6 & [14] & 1 & EX-CCCII & 2 & Yes & No & No & CM & 3.18 \\
\hline \multirow[t]{2}{*}{7} & [15] & 1 & DVCC & 6 & No & No & Yes & CM & - \\
\hline & & 1 & CCII & & & & & & - \\
\hline 8 & [16] & 2 & CCCDTA & 2 & Yes & No & Yes & CM & \\
\hline 9 & [17] & 2 & CDTA & 2 & Yes & No & No & CM & - \\
\hline 10 & [18] & 3 & OTA & 2 & Yes & Yes & No & CM & - \\
\hline \multicolumn{2}{|c|}{ Proposed design } & 2 & VDCC & 4 & Yes & Yes & Yes & $C M$ & 2.27 \\
\hline
\end{tabular}
simultaneously available in any given references. Although the reference no. has the same credentials as designed filter circuit, whereas in Table 4 [21] and [24] are using the same constraints as the proposed design of the oscillator,

Table 3 Comparison of previous published active filter with proposed circuit 
Table 4 Comparison of proposed quadrature with previously reported literature

\begin{tabular}{|c|c|c|c|c|c|c|c|c|c|}
\hline S. no. & Ref. no. & $\begin{array}{l}\text { Name of the } \\
\text { active device }\end{array}$ & $\begin{array}{l}\text { No. of } \\
\text { active } \\
\text { devices }\end{array}$ & $\begin{array}{l}\text { No. of } \\
\text { passive ele- } \\
\text { ments }\end{array}$ & $\begin{array}{l}\text { Utilization } \\
\text { of grounded } \\
\text { passive ele- } \\
\text { ments only }\end{array}$ & $\begin{array}{l}\text { Availability of } \\
\text { Quadrature } \\
\text { outputs }\end{array}$ & $\begin{array}{l}\text { Availability } \\
\text { of explicit } \\
\text { outputs }\end{array}$ & $\begin{array}{l}\text { Availability } \\
\text { of uncoupled } \\
\text { CO and FO }\end{array}$ & $\begin{array}{l}\text { Power } \\
\text { dissipation } \\
(\mathrm{mW})\end{array}$ \\
\hline 1 & {$[21]$} & VDCC & 2 & 4 & Yes & Yes & Yes & Yes & - \\
\hline 2 & {$[22]$} & VDVTA & 1 & 3 & Yes & Yes & No & No & 0.295 \\
\hline 3 & [23] & VDCC & 1 & 4 & Yes & Yes & No & No & - \\
\hline 4 & [24] & VDCC & 2 & 4 & Yes & Yes & Yes & Yes & - \\
\hline 5 & {$[25]$} & VDCC & 1 & 4 & Yes & Yes & Yes & No & 5.6 to 7.2 \\
\hline 6 & {$[26]$} & VDCC & 1 & 3 & Yes & Yes & No & No & 45 \\
\hline 7 & [27] & VDCC & 1 & 4 & Yes & No & No & No & - \\
\hline 8 & [28] & VDCC & 2 & 4 & Yes & Yes & Yes & No & 2.16 \\
\hline 9 & [29] & CDBA & 2 & 5 & No & Yes & No & No & - \\
\hline 10 & [30] & VDCC & 2 & 3 & Yes & No & Yes & No & - \\
\hline 11 & [31] & VDCC & 2 & 4 & No & Yes & Yes & No & - \\
\hline 12 & [32] & DO-CDTA & 2 & 2 & Yes & Yes & Yes & No & - \\
\hline 13 & [33] & VDBA & 2 & 2 & Yes & Yes & Yes & No & - \\
\hline \multicolumn{2}{|c|}{ Proposed design } & VDCC & 2 & 4 & Yes & Yes & Yes & Yes & 1.3 \\
\hline
\end{tabular}

Table 5 Aspect ratios of the CMOS devices [23]

but in all the said references, not all three applications can be generated together from the same circuit topology and moreover the [21] and the presented circuit are two distinguished circuits with different hardware arrangements.

\section{Simulation results}

Necessary simulations, for the verification of the workability of all the designed applications, are presented in this section. CMOS version of VDCC, as shown in Fig. 2 , is used here to perform the simulations. All the CMOS are working in the saturation region of operation. The aspect ratios of the CMOS devices are stated in Table 5 [23]. TSMC $0.18 \mu \mathrm{m}$ process parameters that have been utilized here are taken from [21]. The supply voltage of $\pm 0.9 \mathrm{~V}$ is being used here along with the bias current values of $50 \mu \mathrm{A}$ and $100 \mu \mathrm{A}$ for $I_{B 1}$ and $I_{B 2}$ respectively. The trans-conductance value of both the VDCC is taken as $277.83 \mu \mathrm{A} / \mathrm{V}$.
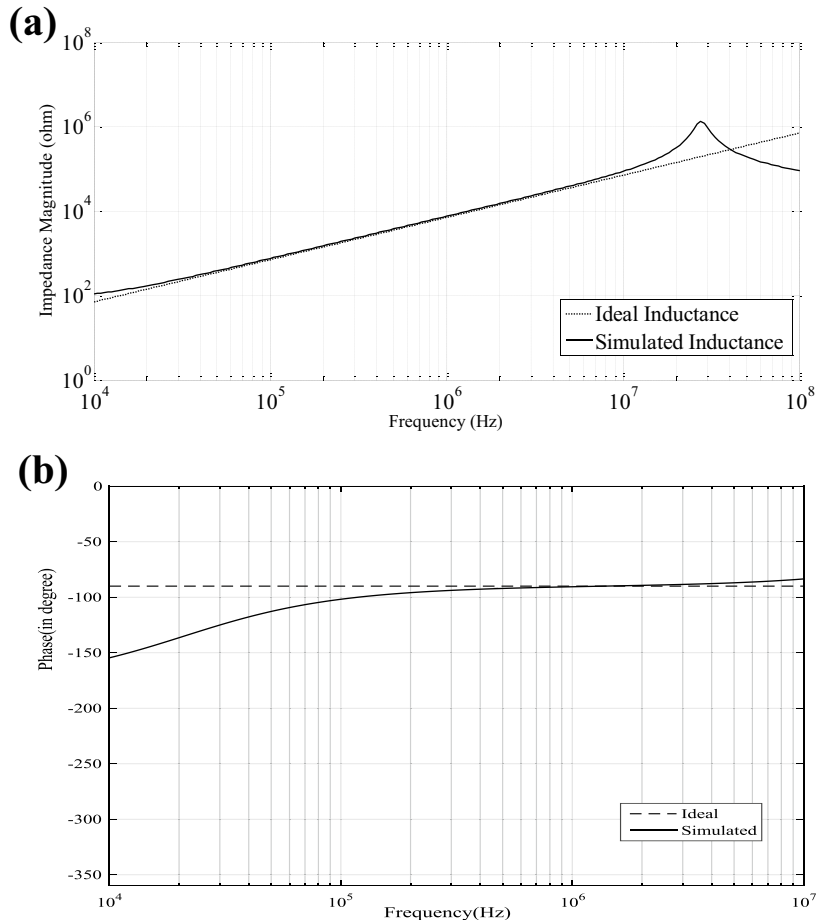

Fig. 9 a Magnitude Response of the VDCC based loss-less grounded inductor. $\mathbf{b}$ Phase response of the VDCC based loss-less grounded inductor

\subsection{Grounded loss-less inductor}

The frequency response, magnitude as well as phase, of the proposed grounded active inductor is shown in Fig. 9. For the attainment of best possible results, the 
passive component values were chosen as $C_{1}=43.8 \mathrm{pF}$ and $R_{1}=3.6 \mathrm{~K}$. The graph depicts that a micro deviation is obtained between the ideal and non-ideal curves of the simulated inductance, up to considerable high frequency, thus strengthening the proposed design idea. The possible reason behind the difference between ideal and simulated values of its inductance's magnitude is the limited frequency range of the used active device.

\subsection{Current mode universal filter}

As described in Sect. 3, the reconfigurable circuit of Fig. 4 also works as a universal filter. The frequency response for the low pass, band pass, high pass, and band stop filter functions are shown in Fig. 10. The passive component values for the simulation of universal filter are taken as $\mathrm{C}_{0}=21.9 \mathrm{pF}$, $\mathrm{C}_{1}=87.6 \mathrm{pF}, \mathrm{R}_{0}=\mathrm{R}_{1}=3.6 \mathrm{~K}$. For the simulation purposes, the central frequency is chosen as $1 \mathrm{MHz}$ and the quality factor is 0.707 . The magnitude as well as phase response of the designed all pass filter is shown in Fig. 11. Here, in Figs. 10 and 11 , the continuous line indicates the outputs achieved by using an ideal block of VDCC and dotted line represents the output generated by using the CMOS version of VDCC.

The slight deviation between ideal and simulated curves is due to the non-ideality and parasitic of the active device. The tunability of angular frequency, for the proposed band pass filter, by keeping its quality factor constant is depicted in Fig. 12. For the same current mode filter, Fig. 13 shows the tunability of the quality factor, for a constant angular frequency. The variation in gain of band pass filter, with constant frequency, is shown in Fig. 14. Tables 6,7 shows the numerical values to support the feasibility of Figs. 12 and 13. Transient response of the derived band pass filter is depicted in Fig. 15. The total harmonic distortion (THD) for the band pass filter is calculated and shown in Fig. 16. It shows that the input current range can be extended up to 100 microamperes with as
Fig. 10 Frequency response of the proposed filter

Fig. 11 Gain and phase response of the designed allpass filter
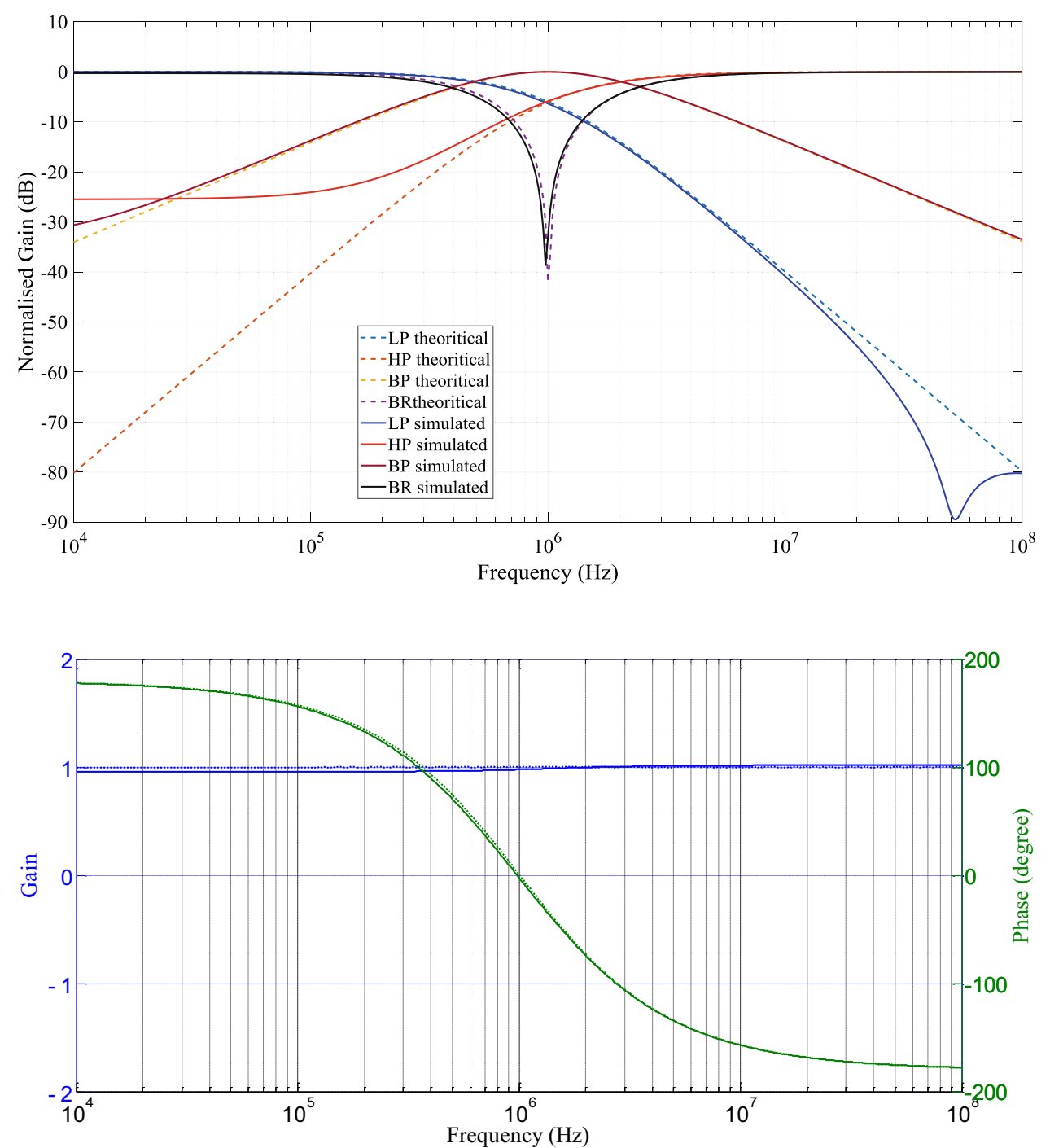

SN Applied Sciences 
Fig. 12 Tunability of the angular frequency for the designed band pass filter

Fig. 13 Tunability of the quality factor for the proposed band pass filter

Fig. 14 Gain variations for the band pass filter with fixed center frequency
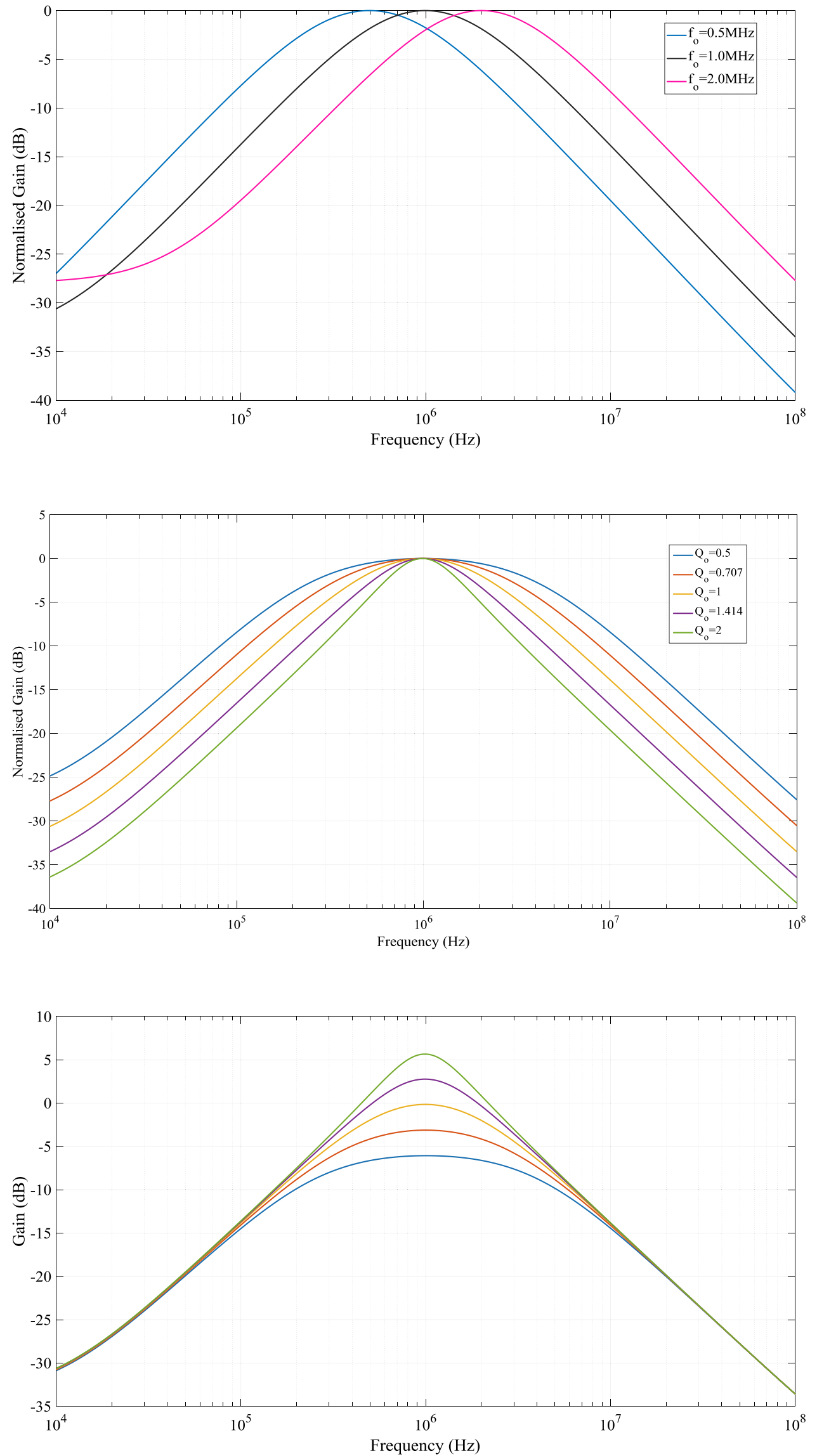
Table 6 Passive components values for Fig. 12

\begin{tabular}{llll}
\hline Frequency (in MHz) & 0.5 & 1 & 2 \\
$\mathrm{R}_{0}$ (in K ohms) & 7.2 & 3.6 & 1.8 \\
$\mathrm{C}_{1}$ (in pF) & 350.4 & 87.6 & 21.9 \\
\hline
\end{tabular}

Table 7 Passive components values for Fig. 13

\begin{tabular}{llllll}
\hline $\mathrm{Q}_{0}$ & 0.5 & 0.707 & 1 & 1.414 & 2 \\
$\mathrm{R}_{0}$ (in K ohms) & 1.8 & 2.54 & 3.6 & 5.09 & 7.2 \\
\hline
\end{tabular}

high as $6 \%$ THD. The statistical analysis, representing the variation in quality factor of $\mathrm{BP}$ response due to tolerances in resistor $\mathrm{R}_{0}$, Monte-Carlo graph is shown in Fig. 17, with 1000 run simulation output. When the noise analysis, of Band Pass filter, for a frequency range starts from $10 \mathrm{kHz}$ to $100 \mathrm{MHz}$ is performed, the input and output magnitudes of noise are found to be equal as $10 \mathrm{e}-29$. It is worth noting here that in Fig. $10 \mathrm{HP}$ response is severely affected by the parasitic calculation because of the cumulative effect of both the filter functions (including non-idealities) i.e., LP and BP will come into the effect [as mentioned in (6)]. The impact of (18) can also be clearly seen in Fig. 12, especially at higher frequencies.

\subsection{Dual mode quadrature oscillator}

A single resistance controlled oscillator can also be realized with the help of Fig. 4, as discussed in Sect. 3. The passive component values, for simulation of quadrature oscillator, are taken as $\mathrm{C}_{0}=\mathrm{C}_{1}=43.8 \mathrm{pF}, \mathrm{R}_{0}=3.6 \mathrm{~K}$. The derived oscillator's transient response, in current mode, is shown in Fig. 18. The steady state, quadrature outputs, in current and in voltage mode are shown in Figs. 19 and 20, respectively. As per Fig. 19, the measured phase difference between two waveforms is $89.14^{\circ}$. Representing the FFT waveform of
Fig. 15 Transient response of the band pass filter

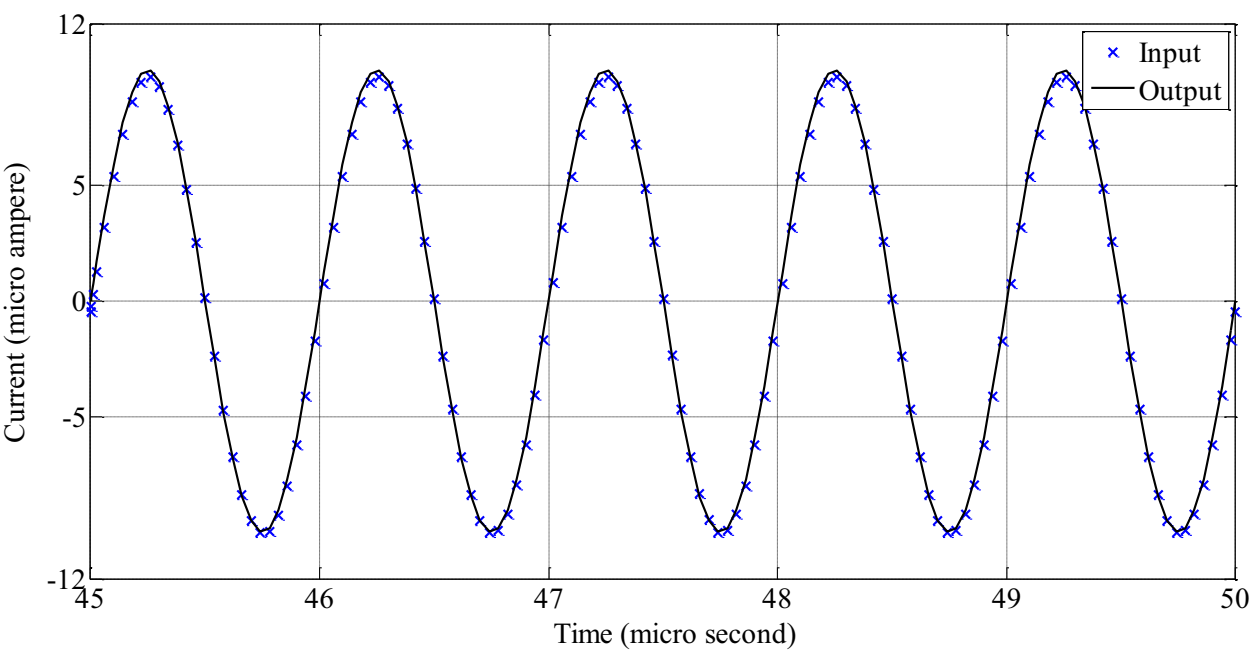

Fig. 16 Total harmonic distortion of the band pass filter

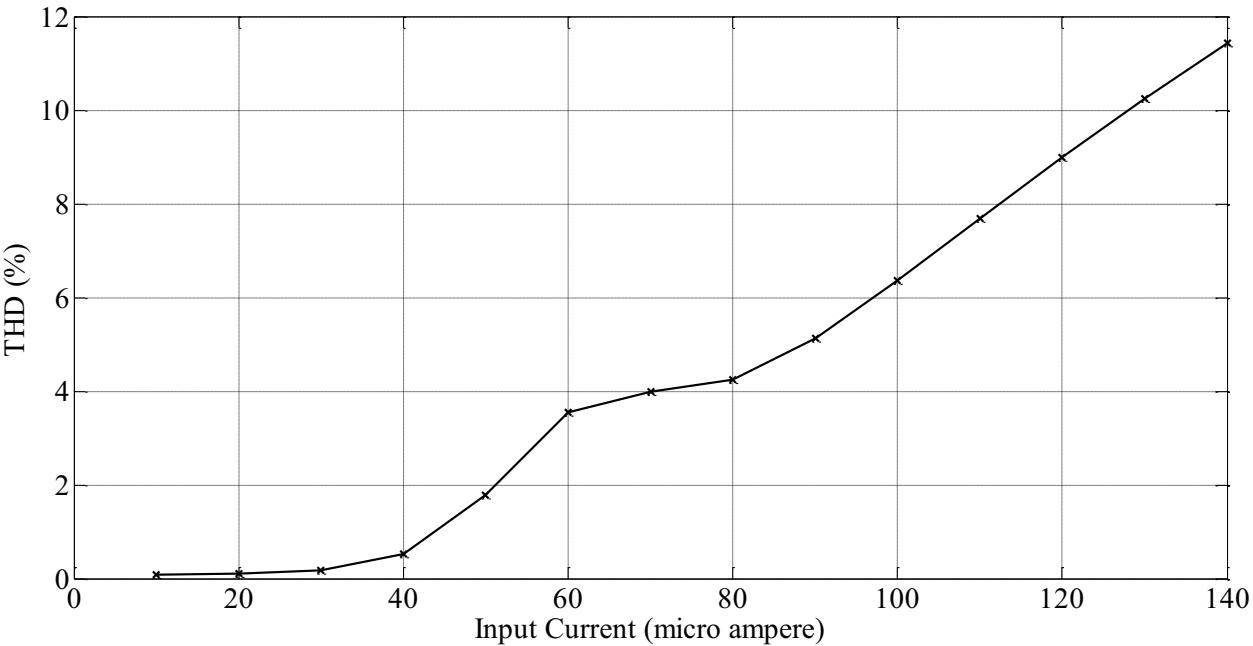



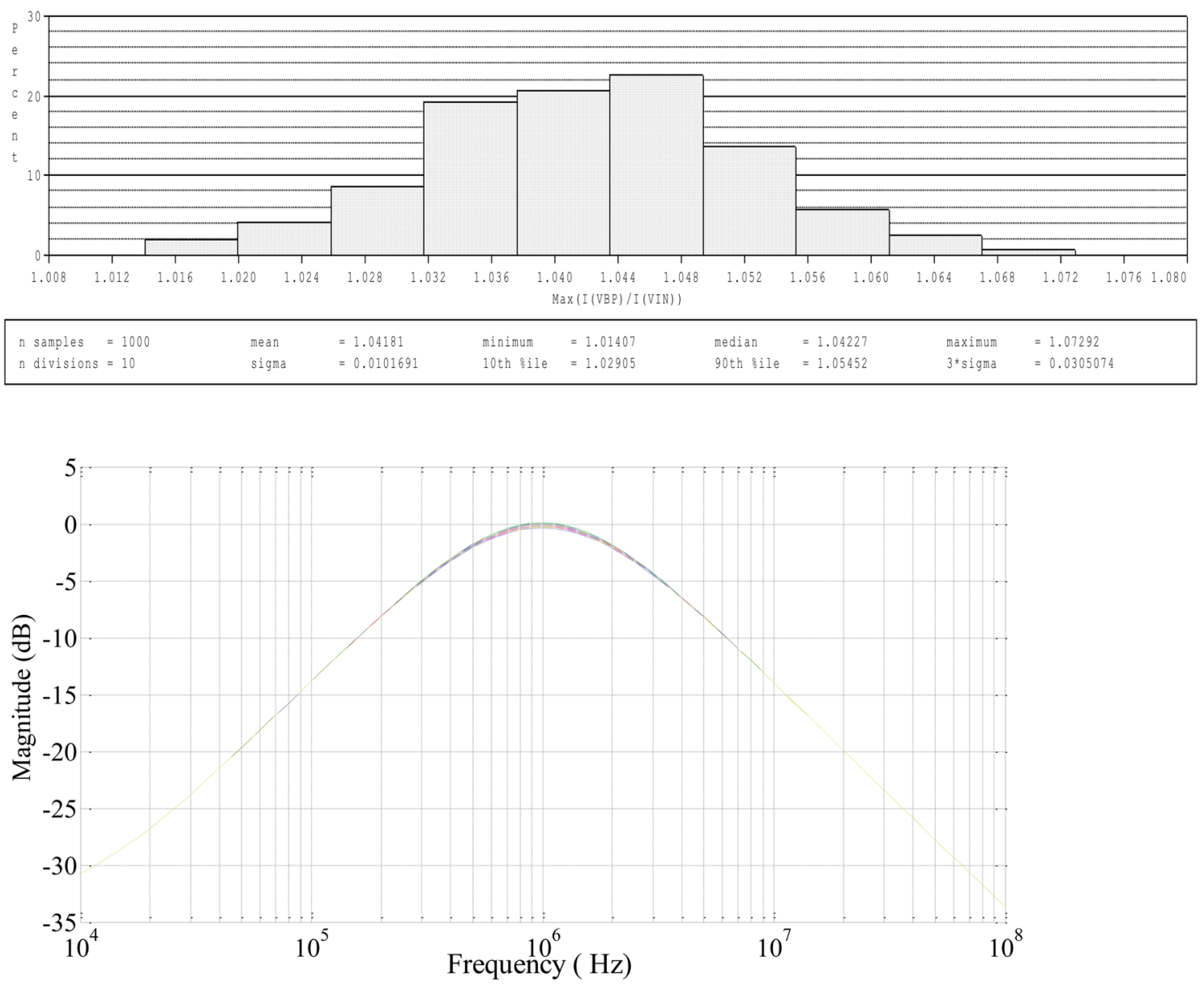

Fig. 17 Monte-Carlo of a band pass filter with a tolerance of $5 \%$ in resistance $R_{0}$

Fig. 18 Transient response of the current mode oscillator

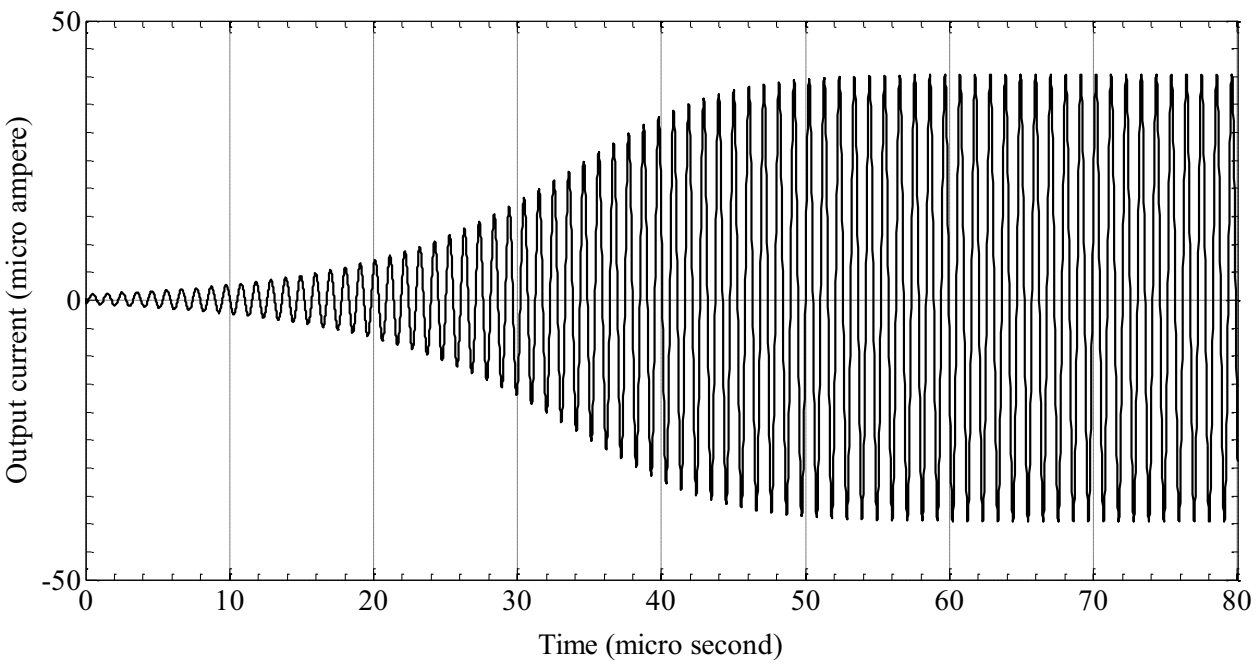

the designed filter, at $1 \mathrm{MHz}$ center frequency, is Fig. 21. To check the bandwidth of the oscillator, $R_{1}$ is swept between a range of values, i.e., from 1 to $50 \mathrm{~K} \Omega$. A set of additional graphical analysis has been provided in Figs. 22, 23, 24, 25 and 26. Here, Fig. 22 shows the percentage of distortion achieved at different oscillation frequencies. When total harmonic distortion of the quadrature oscillator is measured at $1 \mathrm{MHz}$, it was found to be $1.15 \%$ only. The deviation 
Fig. 19 Steady state response of the current mode oscillator

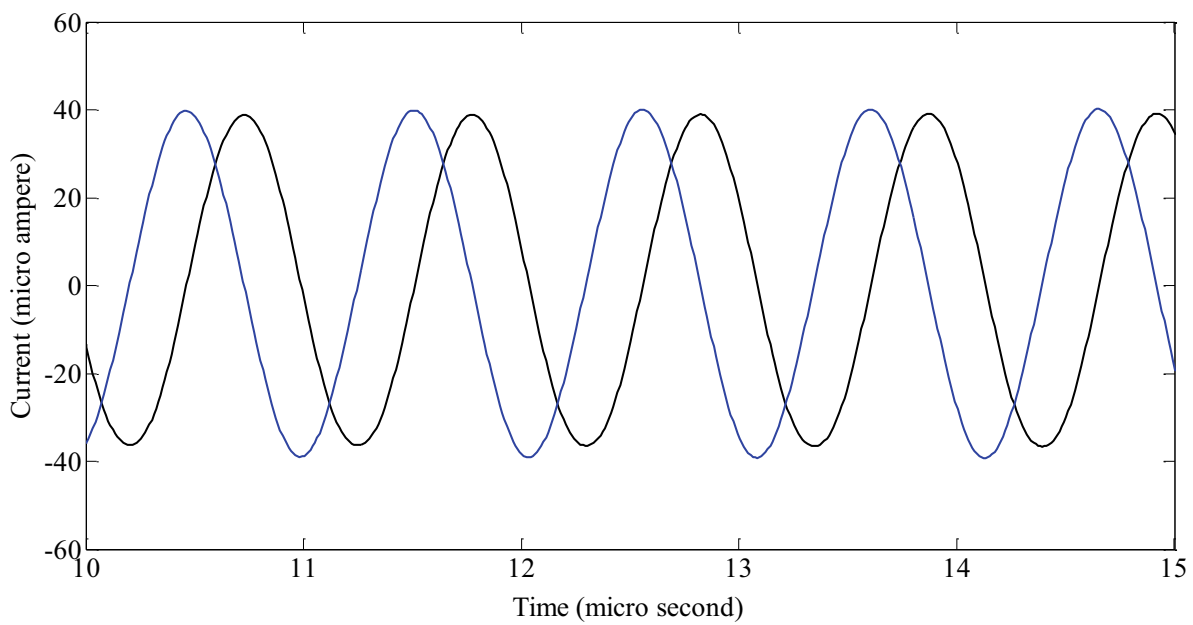

Fig. 20 Transient response of the voltage mode oscillator

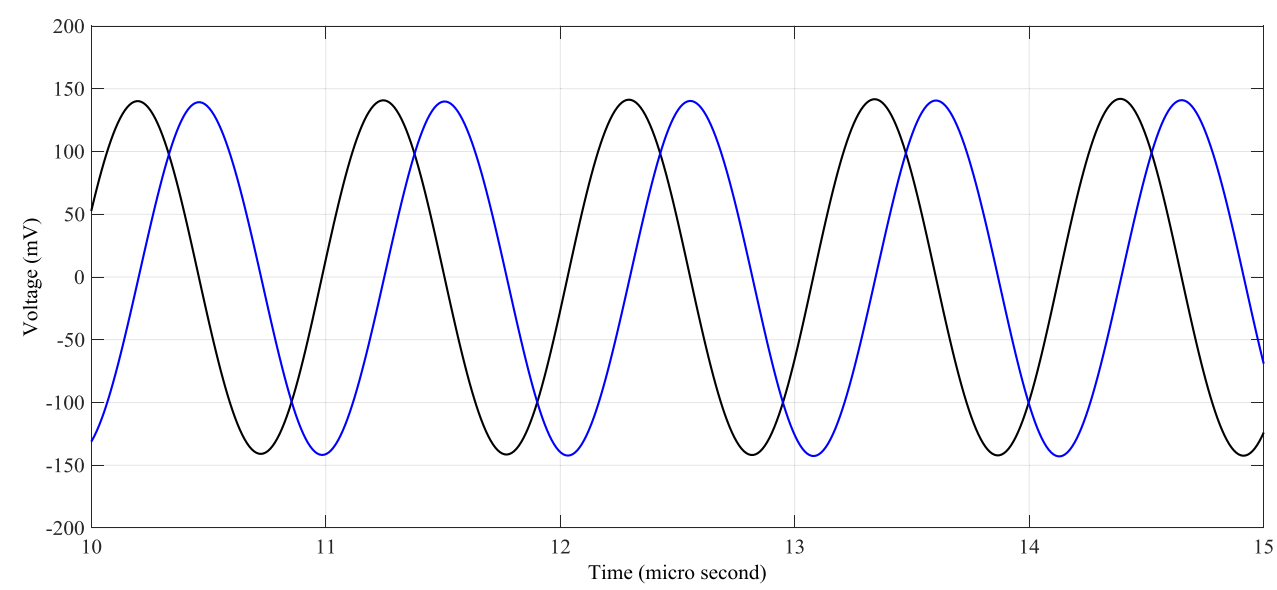

Fig. 21 FFT representation of the centre frequency at $1 \mathrm{MHz}$

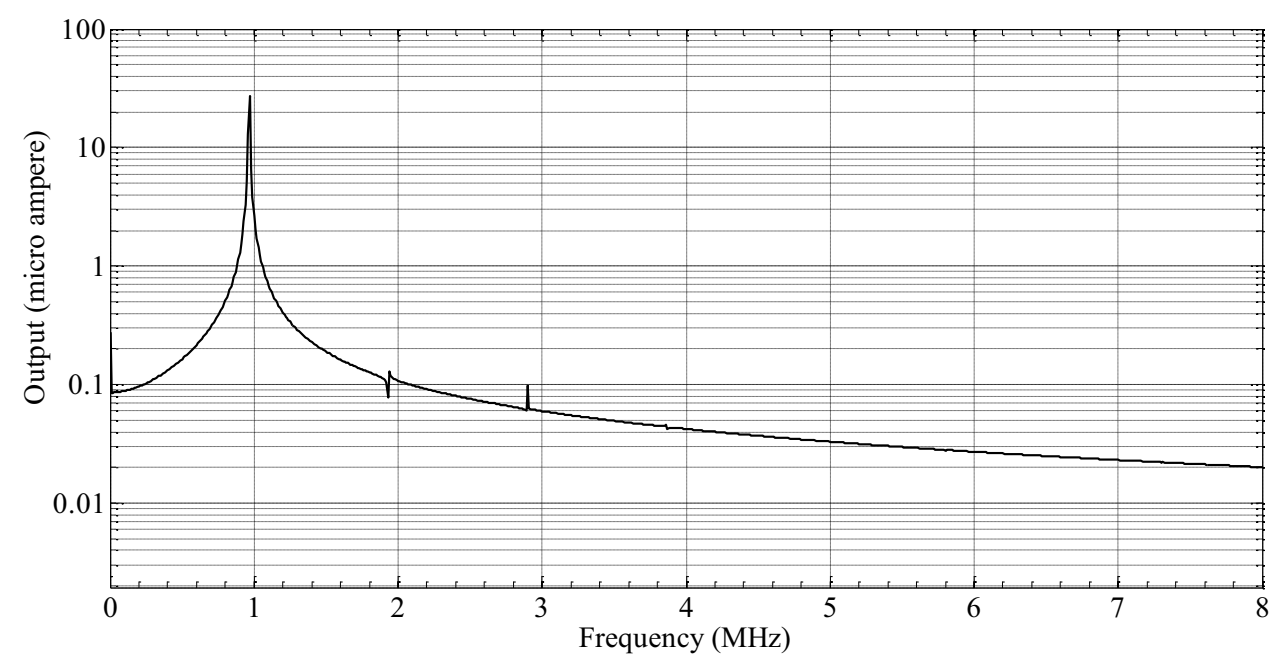

in amplitude of two quadrature current outputs at designated frequencies is represented in Fig. 23. In an oscillator circuit, the amplitude of the output must always be independent of frequency of oscillation. However, Fig. 23 shows the dependency of the amplitude on frequency. In practical this situation is encountered. To deal with it, automatic gain control (AGC) is employed in the circuit. The detailed method of the same is stated in $[35,36]$. Due to brevity of manuscript, this approach has not been explained here and left to readers' discretion. Ideally, the phase difference 
Fig. 22 Percentage of harmonic distortion for the wide range of oscillations

Fig. 23 Variation of output amplitude with respect to oscillation frequency

Fig. 24 Representation of phase angle with respect to oscillation frequency
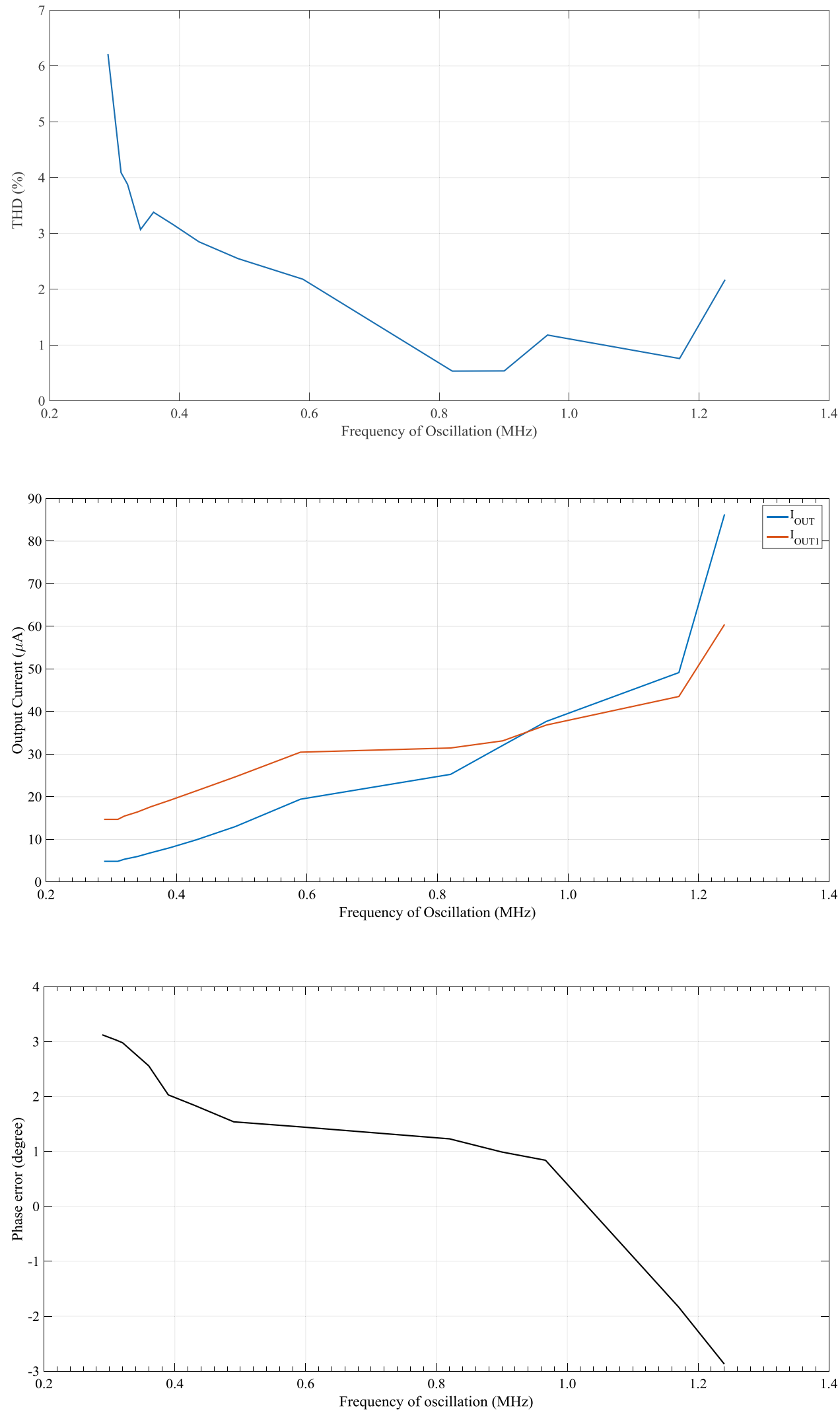
Fig. 25 Variation of $\mathrm{R}_{1}$ with respect to $\mathrm{FO}$ of the designed filter
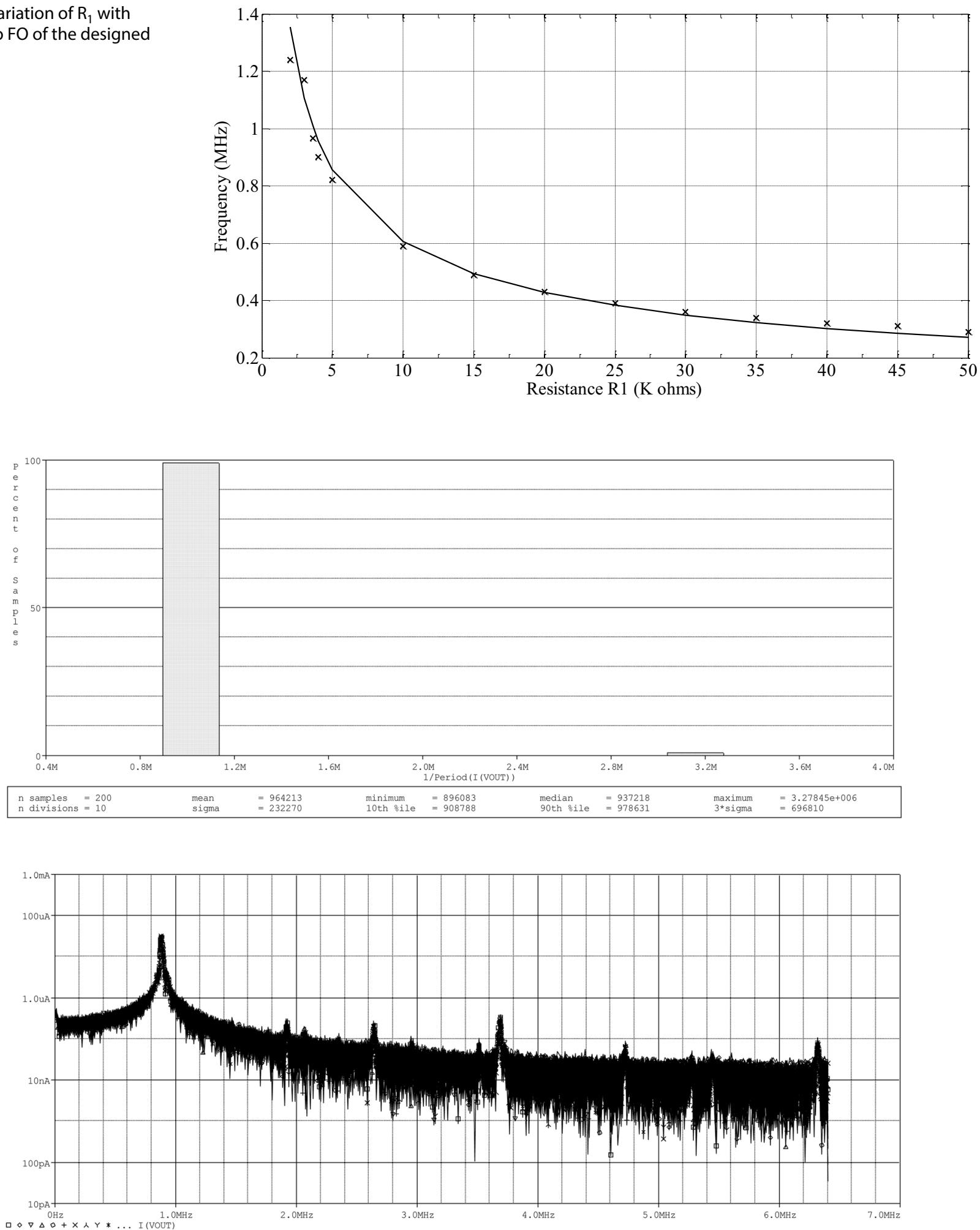

Fig. 26 Monte-Carlo analysis for the proposed oscillator with $0.1 \%$ deviation in resistor $R_{1}$

between two quadrature outputs should be $90^{\circ}$. However, practically the phase difference is slightly different from the expected value. This corresponding phase error has been shown in Fig. 24. Figure 25 shows the wide range of the oscillator. The variation in frequency of oscillation due to 
$0.1 \%$ deviation in grounded resistor $\mathrm{R}_{1}$ is depicted in Fig. 26, through Monte-Carlo analysis with 200 run simulation.

\section{Experimental work}

Before testing the experimental feasibility of the derived circuit it is required that VDCC must be designed with the help of commercially available integrated circuit (IC). There is no single IC that can be replaced by the CMOS model of the device. But VDCC can be realized using the following ICs: (1) by employing LM13700 and AD844 [31] (2) possibly using CA3080 and AD844 (3) utilizing OPA860 only. OPA860 is commonly known as diamond transistor or special purpose amplifier [37]. The approach utilized here is with the help of OPA860, due to the sterling features of this amplifier IC.

1. It is a special function amplifier whose trans-conductance gain can be controlled with the help of single external resistor.

2. The results obtained through OPA860 are close to the actual (derived) results.

3. The bandwidth of this amplifier is very wide.

For the purpose of practical verification, the transistor model of VDCC, i.e., Figure 3, has been used along with some external circuitry. To ensure the validity of hardware arrangements, results obtained through bread board arrangement of proposed sinusoidal oscillator are shown here. It is worth noting that a resistor of $100 \Omega$, in series with the base of the each diamond transistor and in series to buffer, is added [37]. Two keysight programmable DC power supply (E3632A), Caddo $30 \mathrm{MHz}, 2$ channel 4 trace Cathode Ray Oscilloscope (CRO) are used, for the experimentation purposes. The passive element values were chosen as $\mathrm{C}_{0}=\mathrm{C}_{1}=470 \mathrm{pF}, \mathrm{R}_{\mathrm{M0}}=1 / \mathrm{g}_{\mathrm{m} 0}=330 \Omega$, $\mathrm{R}_{\mathrm{M} 1}=1 / \mathrm{g}_{\mathrm{m} 1}=330 \Omega, \mathrm{R}_{0}=0-1 \mathrm{~K} \Omega$ and $\mathrm{R}_{1}=680 \Omega$. The circuit diagram of the hardware realization is shown in Fig. 27. The proposed oscillator has been tested at a frequency of $715 \mathrm{kHz}$ ideally. However, in simulations only $665 \mathrm{kHz}$ was achieved thus giving an error of $6.9 \%$. While practical implementation of the same design gives $645 \mathrm{kHz}$ as oscillation frequency, thus producing an error of 3.01\%, with respect to the simulation frequency.

The wide range of oscillation frequency has been tested practically for the derived quadrature oscillator. Figure 28 shows the steady state response of the quadrature waveforms. The graph presented in Fig. 29 shows the oscillation frequency with respect to the grounded passive resistance value. While testing in the wide range of oscillation frequency from $190 \mathrm{kHz}$ to $1.03 \mathrm{MHz}$, the magnitude of the quadrature outputs are almost constant with less than $1 \%$ deviation in its value. Frequency Response of the designed loss-less grounded inductor implementation using discrete components, i.e., OPA860 has been shown in Fig. 30.

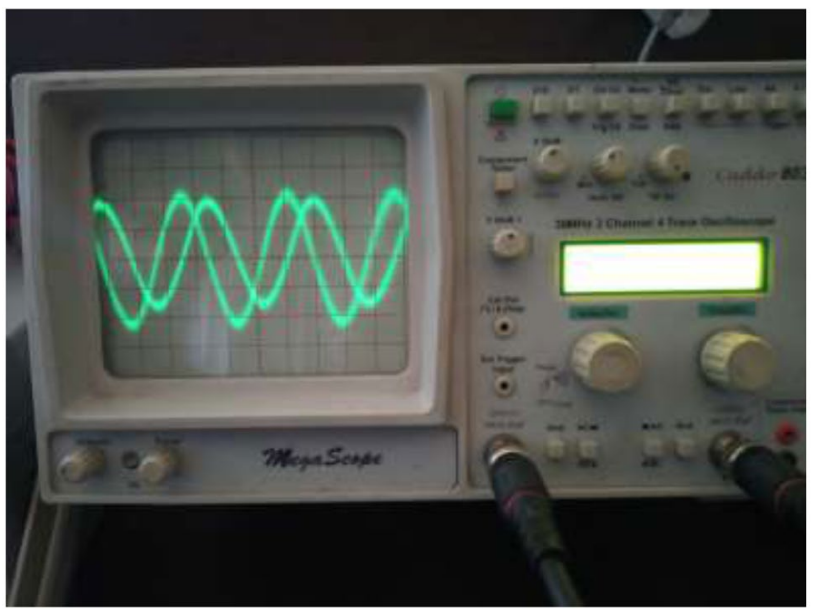

Fig. 28 Steady state response of the quadrature oscillator
Fig. 27 Hardware realization of the proposed quadrature oscillator

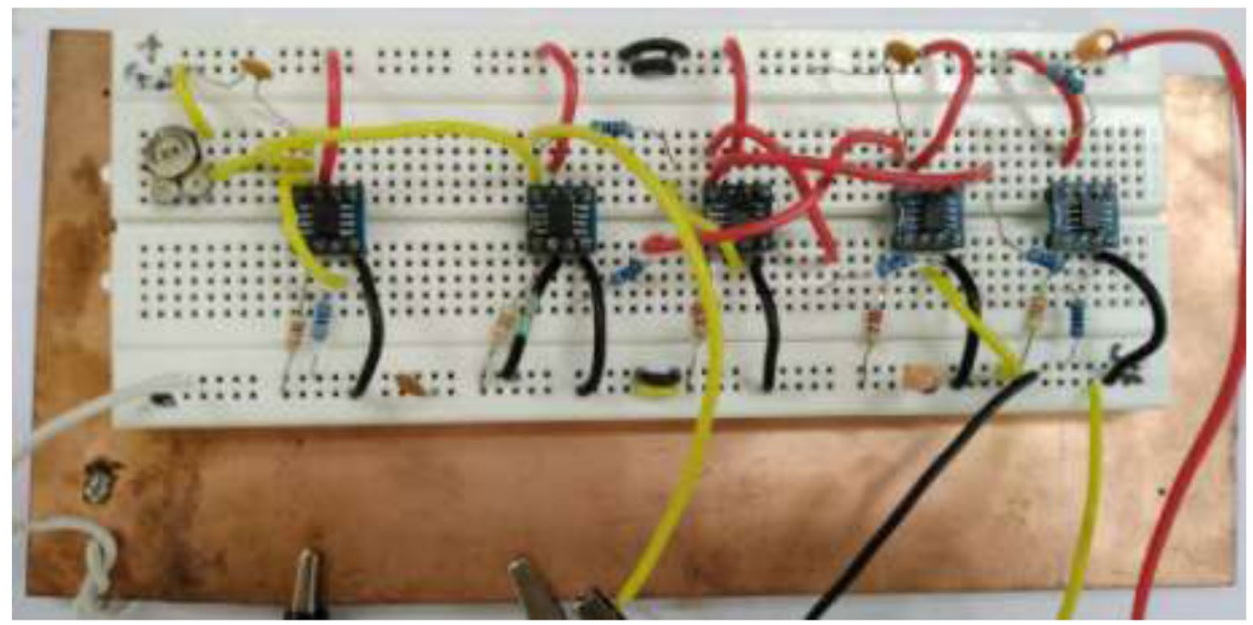


Fig. 29 Variation of oscillation frequency of the designed oscillator
Fig. 30 Frequency Response of the VDCC based loss-less grounded inductor using OPA860
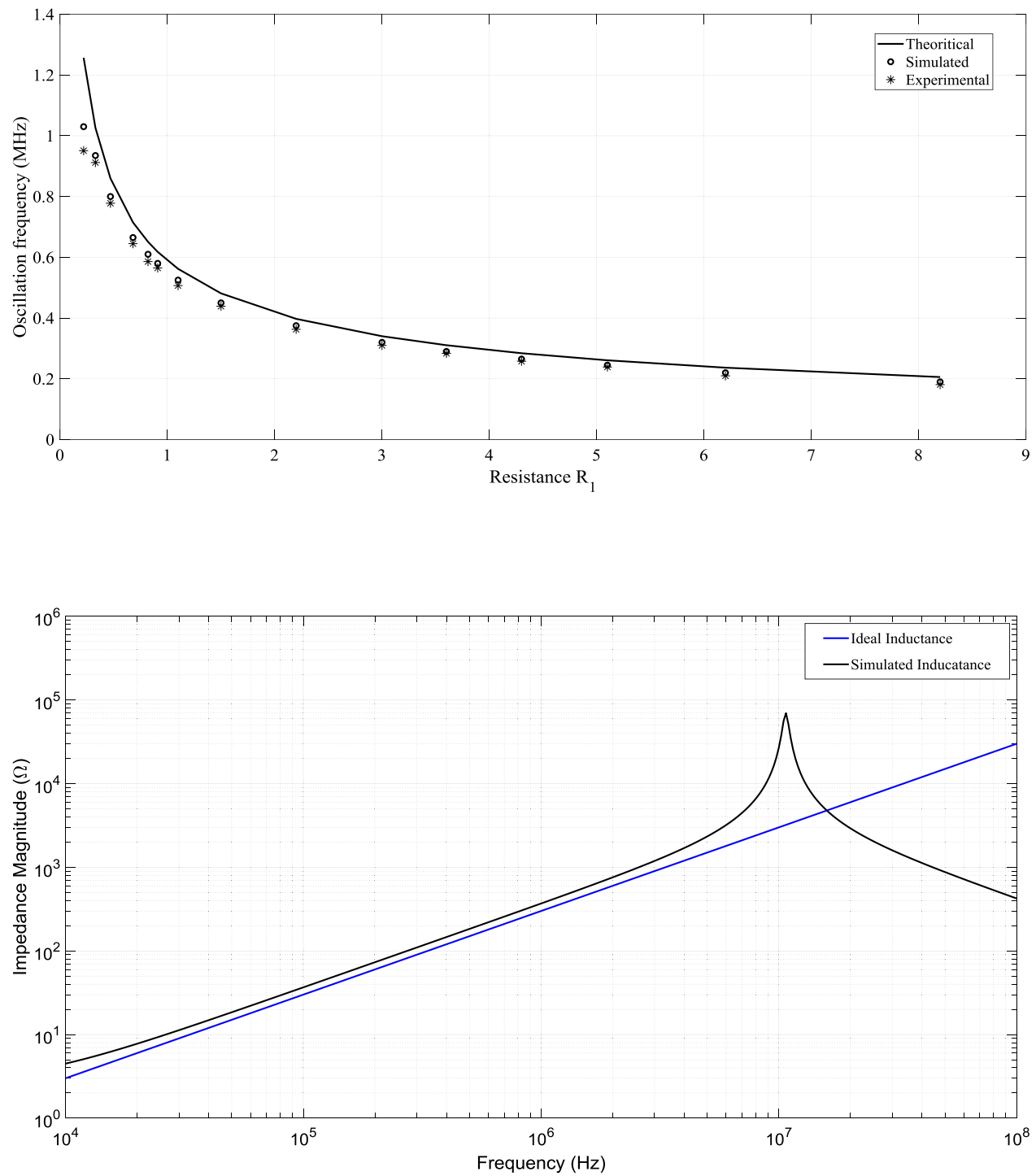

\section{Conclusion}

To present a reconfigurable analog circuit that can be implemented as a synthetic grounded inductor, currentmode universal filter along with a quadrature sinusoidal oscillator employing VDCC and only grounded passive elements was the main purpose of the manuscript. The responses of the derived active filter perform well in the desired frequency except for the HP filter response at low frequencies due to the cumulative parasitic effects. The frequency of oscillation and the condition of oscillation are uncoupled to each other in the case of an oscillator. The derived quadrature oscillator has both explicit currents and voltages outputs availability. THD is less than $6 \%$ in all the usable range of frequency for an oscillator. Different pen-paper analysis and software solutions have been provided here to support the design idea.
Acknowledgements The experimental work has been performed in Analog Electronics Lab at National Institute of Technology Uttarakhand, Srinagar, Uttarakhand. I also acknowledge the efforts and support that has been extended by Ms. Soumya Gupta, Research Intern, at National Institute of Technology Uttarakhand, in preparation of the manuscript.

\section{Compliance with ethical standards}

Conflict of interest The authors declare that they have no competing interests

\section{References}

1. Senani R, Bhaskar DR, Singh AK (2014) Current conveyors: variants, applications and hardware implementations. Springer, Berlin 
2. Senani R, Bhaskar DR, Singh AK, Singh VK (2013) Current feedback operational amplifiers and their applications. Springer, Berlin

3. Tangsrirat W (2017) Synthetic grounded lossy inductance simulators using single VDIBA. IETE J Res 63(1):134-141

4. Yuce E (2009) Novel lossless and lossy grounded inductor simulators consisting of a canonical number of components. Analog Integr Circuits Signal Process 59(1):77-82

5. Prasad D, Bhaskar DR, Singh AK (2010) New grounded and floating simulated inductance circuits using current differencing transconductance Amplifiers. Radio Eng 19(1):194-198

6. Ibrahim MA, Minaei S, Yuce E, Herencsar N, Koton J (2012) Lossy/ lossless floating/grounded inductance simulator using one DDCC. Radio Eng 21(1):2-10

7. Kacar F, Yesil A, Minaei S, Kuntman H (2014) Positive/negative lossy/lossless grounded inductance simulators employing single VDCC and only two passive elements. AEU Int J Electron Commun 68:73-78

8. Kartci A et al (2015) Application possibilities of VDCC in general floating element simulator circuit. In: 2015 European conference on circuit theory and design (ECCTD). IEEE, pp 1-4

9. Abaci A, Yuce E (2017) A new DVCC + based second-order current-mode universal filter consisting of only grounded capacitors. J Circuits Syst Comput 26(09):1750130-18

10. Abdalla KK, Bhaskar DR, Senani R (2012) Configuration for realizing a current mode universal filter and dual-mode quadrature single resistor controlled oscillator. IET Circuits Devices Syst 6(3):159-167

11. Arora TS, Sharma RK (2017) An all-mode KHN equivalent biquad using third generation current conveyor and all grounded passive elements. Proc Natl Acad Sci India Sect A 87(1):97-108

12. Chaturvedi B, Mohan J, Kumar A (2018) A new versatile universal biquad configuration for emerging, signal processing applications. J Circuits Syst Comput 27(12):1850196-18

13. Costas P, Kasimis C, Khateb F (2018) Multiple-input single-output universal biquad filter using single output operational transconductance amplifiers. AEU Int J Electron Commun 93:360-367

14. Agrawal D, Maheshwari S (2017) Current mode filters with reduced complexity using a single EX-CCCII. AEU Int J Electron Commun 80:86-93

15. Horng JW, Wu CM, Herencsar N (2017) Current-mode and transimpedance-mode universal biquadratic filter using two current conveyors. Indian J Eng Mater Sci 24:461-468

16. Jaikla W et al (2013) Electronically tunable current-mode biquad filter employing CCCDTAs and grounded capacitors with low input and high output impedance. AEU Int J Electron Commun 67(12):1005-1009

17. Jin J, Wang C (2014) Current-mode universal filter and quadrature oscillator using CDTAs. Turk J Electr Eng Comput Sci 22(2):276-286

18. Gupta $M$ (2016) New single input multiple output current mode biquad filter using OTAs current mode biquad filter using OTAs. Circuits Syst 7:231-238

19. Arora TS, Rohil B, Gupta S (2019) Fully integrable/cascadable CM universal filter and CM quadrature oscillator using VDCC and only grounded passive elements. J Circuits Syst Comput 28(11):1950181

20. Kacar F, Yesil A, Gurkan K (2015) Design and experiment of VDDC-based voltage mode universal filter. Indian J Pure Appl Phys 53:341-349

21. Gupta M, Arora TS (2017) Realization of current mode universal filter and a dual-mode single resistance controlled quadrature oscillator employing VDCC and only grounded passive elements. Adv Electr Electron Eng 15(5):833-845

22. Alpaslan H (2016) A modified VDVTA and its applications to floating simulators and a quadrature oscillator. Microelectron J 51:1-14

23. Prasad D, Bhaskar DR, Srivastava M (2014) New single VDCCbased explicit current-mode SRCO employing all grounded passive components. Electron J 18(2):81-88

24. Srivastava M, Prasad D (2016) VDCC based dual-mode quadrature sinusoidal oscillator with outputs at appropriate impedance levels. Theor Appl Electr Eng 14:168-177

25. Sotner $R$ et al (2014) Second-order simple multiphase oscillator using z-copy controlled-gain voltage differencing current conveyor. Elektron IR Elektrotech 20(9):13-18

26. Sotner R, Jerabek J, Prokop R, Kledrowetz V (2016) Simple CMOS voltage differencing current conveyor-based electronically tunable quadrature oscillator. Electron Lett 52(12):1016-1018

27. Arora TS (2020) A current-mode single-resistance-controlled oscillator employing VDCC and all grounded passive elements. Iran J Electr Electron Eng 16(2):184-191

28. Gupta M, Dogra P, Arora TS (2019) Novel current mode universal filter and dual-mode quadrature oscillator using VDCC and all grounded passive elements. Aust J Electr Electron Eng 16(4):220-236

29. Arora TS, Gupta S (2018) A new voltage mode quadrature oscillator using grounded capacitors: an application of CDBA. Eng Sci Technol Int J 21(1):43-49

30. Gupta S, Sandhu M, Gupta M, Arora TS (2019) A new electronically tunable CM/VM oscillator using all grounded components. In: Applications of artificial intelligence techniques in engineering. Springer, Singapore, pp 141-151

31. Satipar D, Intani P, Jaikla W (2017) Electronically tunable quadrature sinusoidal oscillator with equal output amplitudes during frequency tuning process. J Electr Comput Eng 2017:1-7

32. Saksit S, Thongsopa C, Jaikla W (2014) Dual-output current differencing transconductance amplifiers-based currentmode sinusoidal quadrature oscillators. J Circuits Syst Comput 23(6):1450084

33. Yeşil A, Kaçar F (2018) Current and voltage mode quadrature oscillator based on voltage differencing buffered amplifier. Electrica 18(1):6-12

34. Biolek D, Senani R, Biolkova V, Kolka Z (2008) Active elements for analog signal processing; classification, review and new proposals. Radio Eng 17(4):15-32

35. Sotner R et al (2012) Study of adjustable gains for control of oscillation frequency and oscillation condition in 3R-2C oscillator. Radio Eng 21(1):392-402

36. Sotner R et al (2014) Precise electronically adjustable oscillator suitable for quadrature signal generation employing active elements with current and voltage gain control. Circuits Syst Signal Process 33(1):1-35

37. OPA860-Wide Bandwidth Operational Transconductance Amplifier (OTA) and Buffer Texas Instruments, SBOS331C-June 2005-Revised August 2008. www.ti.com

Publisher's Note Springer Nature remains neutral with regard to jurisdictional claims in published maps and institutional affiliations. 